Edson de Faria $\cdot$ Welington de Melo

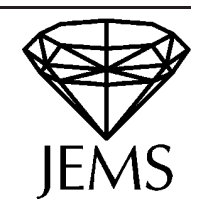

\title{
Rigidity of critical circle mappings I
}

Received November 1, 1998 / final version received July 7, 1999

Abstract. We prove that two $C^{3}$ critical circle maps with the same rotation number in a special set $\mathbb{A}$ are $C^{1+\alpha}$ conjugate for some $\alpha>0$ provided their successive renormalizations converge together at an exponential rate in the $C^{0}$ sense. The set $\mathbb{A}$ has full Lebesgue measure and contains all rotation numbers of bounded type. By contrast, we also give examples of $C^{\infty}$ critical circle maps with the same rotation number that are not $C^{1+\beta}$ conjugate for any $\beta>0$. The class of rotation numbers for which such examples exist contains Diophantine numbers.

\section{Introduction}

The purpose of this paper is to study certain rigidity questions concerning critical circle mappings. This study is continued in [5].

In the qualitative theory of smooth dynamical systems, the notions of rigidity and flexibility play an important role. The smooth systems are usually classified according to the equivalence relation given by topological conjugacies: two smooth maps $f$ and $g$ are topologically equivalent if there exists a homeomorphism $h$ of the ambient space such that $h \circ f=g \circ h$. Such a homeomorphism maps orbits of $f$ onto orbits of $g$. One can also consider a stronger equivalence relation given by smooth conjugacies. This leads to a quantitative or geometric classification of smooth dynamical systems, since a smooth conjugacy, being essentially affine at small scales, preserves the small-scale geometric properties of the dynamics. Hence each topological equivalence class is "foliated" by the smooth conjugacy classes and the quotient space is the moduli or deformation space of the dynamics. The moduli space describes the flexibility of the dynam-

E. de Faria: Instituto de Matemática e Estatística, Universidade de São Paulo, Rua do Matão 1010, Butantã, CEP05508-900 São Paulo SP, Brasil, e-mail: edson@ime.usp.br

W. de Melo: Instituto de Matemática Pura e Aplicada, Estrada Dona Castorina 110, Jardim Botânico, CEP22460 Rio de Janeiro RJ, Brasil, e-mail: demelo@impa.br

This work has been partially supported by the Pronex Project on Dynamical Systems, by FAPESP Grant 95/3187-4 and by CNPq Grant 30.1244/86-3.

Mathematics Subject Classification (1991): 58F03, 58F23 
ics. When this space reduces to a single point, we are in the presence of rigidity.

In general, since eigenvalues at the periodic points are smooth conjugacy invariants, we can hope to find rigidity only in the absence of periodic points. From this viewpoint, the simplest case to consider is that of circle diffeomorphisms. If $f$ is a circle diffeomorphism without periodic points then $f$ is combinatorially equivalent to a rigid rotation $R_{\rho}: x \mapsto x+\rho(\bmod 1)$, in the sense that for each $N$, the first $N$ elements of an orbit of $f$ are ordered in the circle in the same way as the first $N$ elements of an orbit of $R_{\rho}$. From Denjoy's theorem it follows that if $f$ is $C^{2}$ (or at least $C^{1}$ and its derivative has bounded variation) then $f$ is topologically conjugate to $R_{\rho}$. By a fundamental result of Herman [11], improved by Yoccoz [20], if the rotation number $\rho$ satisfies a Diophantine condition such as

$$
\left|\rho-\frac{p}{q}\right| \geq \frac{C}{q^{2+\beta}},
$$

for all rationals $p / q$, with $C>0$ and $0<\beta<1$, and if $f$ is $C^{r}, r \geq 3$, then the conjugacy is $C^{1}$ (it is in fact $C^{r-1-\beta-\varepsilon}$ for every $\varepsilon>0$ ). On the other hand, Arnold proved that some such condition on the rotation number is essential: there exist real analytic circle diffeomorphisms with irrational rotation number such that the conjugacy with a rigid rotation is not even absolutely continuous with respect to Lebesgue measure.

Maps with periodic points cannot be rigid, but we can analyze the rigidity of some relevant invariant set, such as an attractor of the map. This is the situation studied by Sullivan and McMullen in the context of unimodal maps of the interval. They considered the so-called infinitely renormalizable maps of bounded combinatorial type. For such maps, almost all orbits are asymptotic to a Cantor set which is the closure of the critical orbit. They proved that if two such maps are smooth enough and have the same combinatorics then there exists a $C^{1+\alpha}$ diffeomorphism of the real line that conjugates the restriction of the maps to the corresponding Cantor attractors. The tools they developed have been of fundamental importance for the proofs of our results.

Perhaps the most famous rigidity result in Geometry is the celebrated Mostow rigidity theorem. A special case of this theorem states that two compact hyperbolic manifolds of dimension at least 3 which have the same homotopy type are in fact isometric. Here a hyperbolic manifold is the quotient space $\mathbb{H}^{n} / \Gamma$ of the hyperbolic space $\mathbb{H}^{n}$ by a discrete group $\Gamma$ of isometries. The hypothesis of the theorem implies the existence of a quasiconformal homeomorphism of the sphere at infinity that conjugates the actions of the two groups there. Such a-priori step may be regarded as 
a pre-rigidity result. The rigidity is then obtained by proving that this qchomeomorphism is in fact conformal, i.e. a Moebius transformation.

The situation for critical circle mappings fits perfectly into this framework. A critical circle mapping is a homeomorphism $f: S^{1} \rightarrow S^{1}$ that is of class $C^{r}, r \geq 3$, and has a unique critical point $c$ around which, in some $C^{r}$ coordinate system, $f$ has the form $x \mapsto x^{p}$, where $p \geq 3$ is an odd integer called the power law of $f$. Yoccoz proved in [21] that a critical circle mapping without periodic points is topologically conjugate to an irrational rotation. Later, in an unpublished work, he proved that the conjugacy between two critical circle mappings with the same rotation number is in fact quasisymmetric, i.e. there exists a constant $K \geq 1$ such that, for all pairs of adjacent intervals $I_{1}, I_{2}$ of equal length $\left|I_{1}\right|=\left|I_{2}\right|$, we have

$$
\frac{1}{K} \leq \frac{\left|h\left(I_{1}\right)\right|}{\left|h\left(I_{2}\right)\right|} \leq K .
$$

This is in contrast with the diffeomorphism case where, without restriction on the rotation number, the conjugacy may fail to be quasisymmetric (see[16], p. 75). Yoccoz's result, whose proof we present in $\$ 4$ and Appendix $\mathrm{B}$, is the exact analogue of the pre-rigidity step in the proof of Mostow's theorem.

Rigidity Conjecture. If $f, g$ are $C^{3}$ critical circle mappings with the same irrational rotation number of bounded type and the same power-law at the critical point, then there exists a $C^{1+\alpha}$ conjugacy $h$ between $f$ and $g$ for some universal $\alpha>0$.

So far we have succeeded in proving this conjecture only when the maps are real-analytic. Our proof involves real techniques developed in this paper, and deformation of complex structures, developed in [5].

\subsection{Summary of results}

We now present a quick summary of our results. As already mentioned, we prove two main new theorems concerning critical circle homeomorphisms.

The first theorem brings forth the connection between renormalization and rigidity in the context of circle maps. It also indicates that the above Rigidity Conjecture might be true for a much larger class of rotation numbers than bounded type. The proof is given in $\$ 4.4$.

First Main Theorem. There exists a set $\mathbb{A}$ of rotation numbers, having full Lebesgue measure and containing all numbers of bounded type, for which the following holds. Let $f$ and $g$ be topologically conjugate $C^{3}$ critical 
circle maps, and let $h$ be the conjugacy between $f$ and $g$ that maps the critical point of $f$ to the critical point of $g$. If their common rotation number belongs to $\mathbb{A}$, and if their renormalizations converge together exponentially fast in the $C^{0}$-topology, then $h$ is $C^{1+\alpha}$ for some $\alpha>0$.

The second theorem shows that we cannot expect the above Rigidity Conjecture to be true without restriction on the rotation number. The proof occupies $\S 5$ in its entirety.

Second Main Theorem. There exists an uncountable set $\mathbb{B}$ of rotation numbers such that for any $\rho \in \mathbb{B}$ there exist $C^{\infty}$ critical circle maps $f$ and $g$ with rotation number $\rho$ with the property that the conjugacy between $f$ and $g$ sending the critical point of $f$ to the critical point of $g$ is not $C^{1+\beta}$ for any $\beta>0$.

The set $\mathbb{B}$ is very small: its Hausdorff dimension is not greater than $1 / 2$. But it does contain Diophantine numbers, in somewhat remarkable contrast with the case of circle diffeomorphisms. The saddle-node surgery procedure we develop here is quite general, and can be used to produce similar counterexamples to the rigidity of infinitely renormalizable unimodal maps with special unbounded combinatorics.

All estimates performed in this paper rely heavily on the real a-priori bounds of M. Herman [12] and G. Świątek [18]. These bounds are revisited in §3. Several technical consequences of the real bounds needed in this paper, such as the $C^{r-1}$ boundedness of the renormalizations of a $C^{r}$ critical circle map, are gathered in Appendix A.

\section{Preliminaries}

We have three goals in this section. First, we present some of the basic notations commonly used when studying circle maps. Second, we present the notions of commuting pair and renormalization in the context of circle maps, and discuss their relationship. Third, we state the distortion tools that are necessary for proving the real bounds in $\S 3$.

\subsection{Critical circle mappings}

Following the tradition in this subject, we identify the unit circle $S^{1}$ with the one-dimensional torus $\mathbb{R} / \mathbb{Z}$. The obvious advantage of such identification is that it allows us to use additive notation when dealing with circle mappings. 
We briefly recall some standard facts concerning circle mappings. Given a homeomorphism $f: S^{1} \rightarrow S^{1}$, we denote its rotation number by $\rho(f)$. It can be expressed as a continued fraction

$$
\rho(f)=\left[a_{0}, a_{1}, \ldots, a_{n}, \ldots\right]=\frac{1}{a_{0}+\frac{1}{a_{1}+\frac{1}{\frac{\cdots}{a_{n}+\frac{1}{\cdots}}}},}
$$

which can be finite or infinite, depending on whether $\rho(f)$ is rational or irrational, respectively. The positive integers $a_{n}$ are the partial quotients of $\rho(f)$. They give rise to a sequence of return times for $f$, recursively defined by $q_{0}=1, q_{1}=a_{0}$ and $q_{n+1}=a_{n} q_{n}+q_{n-1}$ for all $n \geq 1$ (for which $a_{n}$ exists - an assumption that will be implicit henceforth). Given $x \in S^{1}$ and $n \geq 1$, we denote by $J_{n}(x)$ the closed interval containing $x$ whose endpoints are $f^{q_{n}}(x)$ and $f^{q_{n-1}}(x)$. We also let $I_{n-1}(x) \subseteq J_{n}(x)$ be the closed interval whose endpoints are $x$ and $f^{q_{n-1}}(x)$. Observe that $J_{n}(x)=I_{n}(x) \cup I_{n-1}(x)$ for all $n \geq 1$.

From the dynamics standpoint, we are not interested in all circle homeomorphisms, but only in those that possess a unique critical point in $S^{1}$, being local diffeomorphisms everywhere else. These are the so-called critical circle maps. More precisely, let $f: S^{1} \rightarrow S^{1}$ be a $C^{r}$ homeomorphism, for some $r \geq 1$. We say that $f$ is a critical circle map if there exists $c \in S^{1}$ (the critical point) such that $f^{\prime}(c)=0$ and $f^{\prime}(x) \neq 0$ for all $x \neq c$. Moreover, we require $f$ to have a power-law at $c$. This means that in a suitable $C^{r}$ coordinate system around the critical point, our $f$ is represented by a map of the form $x \mapsto x|x|^{p-1}+a$, for some real number $p>1$ called the power-law exponent of $f$. The class of all $C^{r}$ critical circle maps will be denoted by $\operatorname{Crit}^{r}\left(S^{1}\right)$.

Since the critical point $c$ of a critical circle map is a distinguished point on the circle, we will write $I_{n}$ and $J_{n}$ throughout, instead of $I_{n}(c)$ and $J_{n}(c)$, respectively.

\subsection{Commuting pairs}

We will study the successive renormalizations of a critical circle map $f$. Here, as in many other settings in dynamics, the word renormalization is taken to mean a (suitably normalized) Poincaré first return map of $f$ to some neighborhood of its critical point. Abstracting the essential features of such first return maps yields the notion of commuting pair, due to O. Lanford [13] and D. Rand [17]. We formulate this notion as follows. 
Definition. A $C^{r}$ commuting pair consists of two mappings $\boldsymbol{f}_{-}:[\lambda, 0] \rightarrow \mathbb{R}$, where $\lambda<0$, and $\boldsymbol{f}_{+}:[0,1] \rightarrow \mathbb{R}$, satisfying the following conditions.

[P $\mathrm{P}_{1}$ Both $\boldsymbol{f}_{-}$and $\boldsymbol{f}_{+}$are $C^{r}$ orientation-preserving homeomorphisms onto their images.

$\left[\mathrm{P}_{2}\right]$ We have $\boldsymbol{f}_{-}(0)=1, \boldsymbol{f}_{+}(0)=\lambda$ and $0<\boldsymbol{f}_{-}(\lambda)=\boldsymbol{f}_{+}(1)<1$.

$\left[\mathrm{P}_{3}\right]$ We have $D \boldsymbol{f}_{-}(x)>0$ for all $\lambda \leq x<0$, and $D \boldsymbol{f}_{+}(x)>0$ for all $0<x \leq 1$

$\left[\mathrm{P}_{4}\right] \quad$ For each $1 \leq k \leq r$, we have $D^{k}\left(\boldsymbol{f}_{+} \circ \boldsymbol{f}_{-}\right)(0)=D^{k}\left(\boldsymbol{f}_{-} \circ \boldsymbol{f}_{+}\right)(0)$.

A critical commuting pair is a commuting pair such that $D f_{-}(0)=0=$ $D \boldsymbol{f}_{+}(0)$.

Although it is more customary to use the symbols $\xi$ and $\eta$ instead of $\boldsymbol{f}_{-}$ and $f_{+}$, respectively, the present notation will be more convenient for our purposes in this paper. It can be proved that, in the presence of the other conditions, $\mathrm{P}_{4}$ is equivalent to the following.

$\left[\mathrm{P}_{4}^{\prime}\right]$ There exist open intervals $\Delta_{-} \supseteq[\lambda, 0]$ and $\Delta_{+} \supseteq[0,1]$, and $C^{r}$ homeomorphic extensions $\boldsymbol{F}_{-}: \Delta_{-} \rightarrow \mathbb{R}$ and $\boldsymbol{F}_{+}: \Delta_{+} \rightarrow \mathbb{R}$ of $\boldsymbol{f}_{-}$and $\boldsymbol{f}_{+}$respectively, satisfying $\boldsymbol{F}_{+} \circ \boldsymbol{F}_{-}(x)=\boldsymbol{F}_{-} \circ \boldsymbol{F}_{+}(x)$ for all $x \in \Delta_{-} \cap \Delta_{+}$such that $\boldsymbol{F}_{ \pm}(x) \in \Delta_{\mp}$ (the set of such $x$ is an open interval around 0 ).

This justifies the name commuting pair. The class of all $C^{r}$ critical commuting pairs will be denoted by $\mathbf{P}^{r}$. We shall henceforth identify a commuting pair $\left(\boldsymbol{f}_{-}, \boldsymbol{f}_{+}\right)$with a single map $\boldsymbol{f}:[\lambda, 1] \rightarrow[\lambda, 1]$, called the shadow of the commuting pair, defined as follows,

$$
f(x)= \begin{cases}f_{-}(x), & \text { when } \lambda \leq x \leq 0 \\ f_{+}(x), & \text { when } 0<x \leq 1 .\end{cases}
$$

To each commuting pair $f$ we associate an element $a \in \mathbb{N} \cup\{\infty\}$ called the height of $f$, in the following way. If there exists $n \geq 1$ such that $f^{n+1}(1)<0 \leq f^{n}(1)$, then we set $a=n$; otherwise we set $a=\infty$. It is clear that $f$ has infinite height if and only if there exists $0<\bar{x}<1$ such that $f(\bar{x})=\bar{x}$.

\subsection{Renormalizing a commuting pair}

Every commuting pair $f$ with finite height $a$ such that $f^{a}(1)>0$ can be renormalized, in the following sense. Let $\Lambda: \mathbb{R} \rightarrow \mathbb{R}$ be the linear map 
$x \mapsto \lambda x$, let $\lambda^{\prime}=f^{a}(1) / \lambda<0$, and let $\mathcal{R} f:\left[\lambda^{\prime}, 1\right] \rightarrow\left[\lambda^{\prime}, 1\right]$ be the map defined by

$$
\mathcal{R} f(x)= \begin{cases}\Lambda^{-1} \circ f \circ \Lambda(x), & \text { when } \lambda^{\prime} \leq x \leq 0 \\ \Lambda^{-1} \circ f^{a+1} \circ \Lambda(x), & \text { when } 0<x \leq 1 .\end{cases}
$$

This map is (the shadow of) a commuting pair $\left(\mathcal{R} \boldsymbol{f}_{-}, \mathcal{R} \boldsymbol{f}_{+}\right)$, called the first renormalization of $f$. Equivalently,

$$
\begin{cases}\mathcal{R} \boldsymbol{f}_{-}(x)=\Lambda^{-1} \circ \boldsymbol{f}_{+} \circ \Lambda(x), & \text { for all } \lambda^{\prime} \leq x \leq 0 \\ \mathcal{R} \boldsymbol{f}_{+}(x)=\Lambda^{-1} \circ \boldsymbol{f}_{+}^{a} \circ \boldsymbol{f}_{-} \circ \Lambda(x), & \text { for all } 0 \leq x \leq 1 .\end{cases}
$$

The class of all $C^{r}$ critical commuting pairs which are renormalizable in this sense will be denoted $\mathbf{P}_{1}^{r}$. In this way, we have a well-defined map $\mathcal{R}: \mathbf{P}_{1}^{r} \rightarrow \mathbf{P}^{r}$, the so-called renormalization operator. More generally, for all $n \geq 1$ we write $\mathbf{P}_{n}^{r}=\mathcal{R}^{-n}\left(\mathbf{P}^{r}\right)$ for the set of all $C^{r}$ critical commuting pairs which can be renormalized $n$ times. We have $\mathbf{P}_{n+1}^{r} \subseteq \mathbf{P}_{n}^{r}$ for all $n$. We are especially interested in the set of all infinitely renormalizable critical commuting pairs, namely

$$
\mathbf{P}_{\infty}^{r}=\bigcap_{n \geq 1} \mathbf{P}_{n}^{r}
$$

Given $\boldsymbol{f} \in \mathbf{P}^{r}$, let $a_{0}=a$ be its height, and for each $n \geq 1$ such that $\boldsymbol{f} \in \mathbf{P}_{n}^{r}$, let $a_{n}$ be the height of $\mathcal{R}^{n}(\boldsymbol{f})$. This can be a finite or infinite sequence; in any case, using the convention $1 / \infty=0$, we define the rotation number of $f$ to be

$$
\rho(\boldsymbol{f})=\left[a_{0}, a_{1}, \ldots, a_{n}, \ldots\right]=\frac{1}{a_{0}+\frac{1}{a_{1}+\frac{1}{\frac{\cdots}{a_{n}+\frac{1}{\ldots}}}} .}
$$

In particular, $\rho(\mathcal{R} \boldsymbol{f})=\left[a_{1}, a_{2}, \ldots\right]$, that is, the renormalization operator acts as the Gaussian shift on continued fractions.

\subsection{Renormalizing a critical circle map}

Let $f$ be a critical circle map with critical point $c$, and for each $k \geq 0$ such that $f^{q_{k}}(c) \neq c$, let $A_{k}: \mathbb{R} \rightarrow \mathbb{R} / \mathbb{Z}$ be the affine covering map such that $A_{k}([0,1])=I_{k}$, with $A_{k}(0)=c$ and $A_{k}(1)=f^{q_{k}}(c)$. For each $n \geq 1$ such 
that $f^{q_{k}}(c) \neq c$ for all $0 \leq k \leq n$, consider the Poincaré first return map $f_{n}: I_{n} \cup I_{n-1} \rightarrow I_{n} \cup I_{n-1}$, namely

$$
f_{n}(x)= \begin{cases}f^{q}(x) & \text { when } x \in I_{n} \\ f^{Q}(x) & \text { when } x \in I_{n-1},\end{cases}
$$

where $q=q_{n-1}$ and $Q=q_{n}$. Define $\lambda_{n}$ to be the largest negative number such that $A_{n-1}\left(\lambda_{n}\right)=f^{Q}(c)$ (one sees in fact that $\left.\lambda_{n}=-\left|I_{n}\right| /\left|I_{n-1}\right|\right)$. Then $A_{n-1}\left(\left[\lambda_{n}, 0\right]\right)=I_{n}$ and $A_{n-1}([0,1])=I_{n-1}$, and we can consider the map $\boldsymbol{f}_{n}:\left[\lambda_{n}, 1\right] \rightarrow\left[\lambda_{n}, 1\right]$ given by $\boldsymbol{f}_{n}=A_{n-1}^{-1} \circ f_{n} \circ A_{n-1}$. Here, it is implicit that $A_{n-1}^{-1}$ is the inverse branch that maps $I_{n} \cup I_{n-1}$ onto $\left[\lambda_{n}, 1\right]$. This defines (the shadow of) a $C^{r}$ critical commuting pair called $n$-th renormalization of $f$. It is well-defined provided the rotation number of $f$ has a continuedfraction development of length at least $n+1$ (in particular it is well-defined for all $n$ when the rotation number of $f$ is irrational). It is easy to see in this case that $\boldsymbol{f}_{k+1}=\mathcal{R} \boldsymbol{f}_{k}$ for all $1 \leq k \leq n-1$. Moreover, if $a_{0}, a_{1}, \ldots$ are the partial quotients of the rotation number of $f$ then, from the recurrence relations satisfied by the sequence of return times $q_{k}$, we see at once that the height of $\boldsymbol{f}_{k}$ is equal to $a_{k}$, and that $\rho\left(\boldsymbol{f}_{k}\right)=\left[a_{k}, a_{k+1}, \ldots\right]$.

Remark. Note that the largest interval containing $I_{n}$ on which $f^{q_{n-1}}$ is a diffeomorphism is $\left[\alpha_{n}, c\right]$ where $f^{a_{n-1} q_{n-1}}\left(\alpha_{n}\right)=f^{q_{n}}(c)$, that is, $\alpha_{n}=$ $f^{q_{n-2}}(c)$. Similarly, the largest interval containing $I_{n-1}$ on which $f^{q_{n}}$ is a diffeomorphism is $\left[c, \beta_{n}\right]$ where $f^{q_{n}}\left(\beta_{n}\right)=f^{q_{n-1}}(c)$.

\subsection{The $C^{k}$ metrics}

The following is only one of several equivalent ways of defining a $C^{k}$ distance between commuting pairs. We normalize our commuting pairs to be defined on $[0,1]$, using for each $f$ a fractional linear transformation that maps $\lambda, 0,1$ respectively to $0, \frac{1}{2}, 1$, and then use the $C^{k}$ norm of the difference of the normalized pairs. The $C^{k}$ distance between $\boldsymbol{f}$ and $\boldsymbol{g}$ defined in this fashion is denoted by $d_{k}(\boldsymbol{f}, \boldsymbol{g})$.

Let us be a bit more precise. If a function $\varphi:[0,1] \rightarrow \mathbb{R}$ has a jump discontinuity at $x=1 / 2$ but is elsewhere $k$ times continuously differentiable, let $\|\varphi\|_{k}=\max \left\{\left\|\varphi^{-}\right\|_{k},\left\|\varphi^{+}\right\|_{k}\right\}$ where $\varphi^{-}$is the restriction of $\varphi$ to $\left[0, \frac{1}{2}\right]$ and $\varphi^{+}$is the restriction of $\varphi$ to $\left[\frac{1}{2}, 1\right]$. Given two elements $f:[\lambda, 1] \rightarrow \mathbb{R}$ and $\boldsymbol{g}:[\mu, 1] \rightarrow \mathbb{R}$ of $\mathbf{P}^{r}$, and given $0 \leq k \leq r$, let $A_{\lambda}$ be the fractional linear transformation which maps $0, \lambda, 1$ to $0, \frac{1}{2}, 1$, respectively, and let $A_{\mu}$ be similarly defined. Then write

$$
d_{k}(\boldsymbol{f}, \boldsymbol{g})=\max \left\{|\lambda-\mu|,\left\|A_{\lambda} \boldsymbol{f} A_{\lambda}^{-1}-A_{\mu} \boldsymbol{g} A_{\mu}^{-1}\right\|_{k}\right\} .
$$




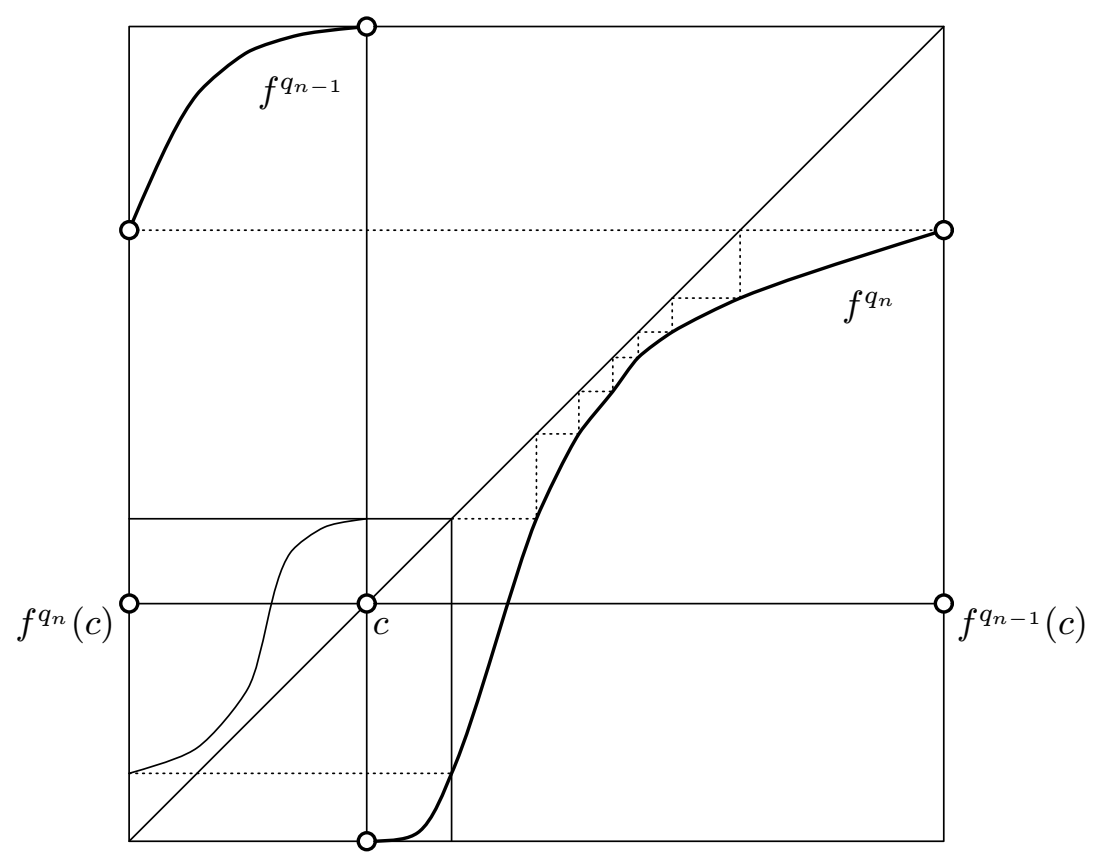

Fig. 1. Two consecutive renormalizations of $f$

Lemma 2.1. For each $0 \leq k \leq r, d_{k}$ is a metric.

Proof. The only thing not entirely obvious is that $d_{k}(\boldsymbol{f}, \boldsymbol{g})=0$ implies $\boldsymbol{f}=\boldsymbol{g}$. But if $d_{k}(\boldsymbol{f}, \boldsymbol{g})=0$ then on one hand $\lambda=\mu$, so that $A_{\lambda}=A_{\mu}$, and on the other hand $A_{\lambda} \boldsymbol{f} A_{\lambda}^{-1}-A_{\mu} \boldsymbol{g} A_{\mu}^{-1}=0$, so that $\boldsymbol{f}=A_{\lambda}^{-1} A_{\mu} \boldsymbol{g} A_{\mu}^{-1} A_{\lambda}=\boldsymbol{g}$.

Proposition 2.2. Let $\boldsymbol{f}:[\lambda, 1] \rightarrow[\lambda, 1]$ and $\boldsymbol{g}:[\mu, 1] \rightarrow[\mu, 1]$ be elements of $\mathbf{P}_{\infty}^{r}$, and suppose there exists a $C^{r}$ diffeomorphism $h:[\lambda, 1] \rightarrow[\mu, 1]$ such that $h \circ f=g \circ h$. Then for all $k \leq r-1$ the distances $d_{k}\left(\mathcal{R}^{n}(\boldsymbol{f}), \mathcal{R}^{n}(\boldsymbol{g})\right)$ converge to 0 at an exponential rate.

Proof. Let $\boldsymbol{f}_{n}=\mathcal{R}^{n} \boldsymbol{f}$ and $\boldsymbol{g}_{n}=\mathcal{R}^{n} \boldsymbol{g}$. Then $\boldsymbol{f}_{n}=h_{n}^{-1} \circ \boldsymbol{g}_{n} \circ h_{n}$, where $h_{n}$ is obtained from $h$ by restriction and affine rescaling. We will see below (after we prove the real bounds for critical circle maps, $c f$. Theorem 3.1) that $\left\{h_{n}\right\}$ converges exponentially in the $C^{r}$ sense to the space of affine maps. Therefore, we have that $d_{r-1}\left(\boldsymbol{f}_{n}, \boldsymbol{g}_{n}\right) \rightarrow 0$ exponentially fast.

\subsection{Distortion tools}

In $\S 3$ we will need some distortion tools to get real bounds for critical circle maps. The most basic is the notion of cross-ratio distortion. Given intervals 
$M \subseteq T$ on the line or circle, their cross-ratio is defined as

$$
D(M, T)=\frac{|M||T|}{|L||R|},
$$

where $L$ and $R$ are the left and right components of $T \backslash M$. The cross-ratio distortion of a map $f$ (whose domain contains $T$ ) on the pair of intervals $(M, T)$ is

$$
B(f ; M, T)=\frac{D(f(M), f(T))}{D(M, T)} .
$$

Cross-ratios are always increased by a map with negative Schwarzian derivative. More precisely, if $f$ is $C^{3}$ and $S f<0$ then $B(f ; M, T)>1$.

Lemma 2.3. (Cross-ratio distortion principle)

Given a map $f$ as above, $m \geq 1$ and intervals $M \subseteq T$ such that $f^{m} \mid T$ is a diffeomorphism onto its image, we have

$$
B\left(f^{m} ; M, T\right) \geq \exp \left\{-\sigma \sum_{j=0}^{m-1}\left|f^{j}(T)\right|\right\},
$$

where $\sigma>0$ depends on $f$ and $\max _{0 \leq j \leq m-1}\left|f^{j}(T)\right|$.

For a proof of (a much more general version of) this principle, see [16], p. 287. This fact will be used in combination with the following classical distortion principle. For intervals $M \subseteq T$ as above we define the space of $M$ inside $T$ to be the smallest of the ratios $|L| /|M|$ and $|R| /|M|$.

Lemma 2.4. (Koebe distortion principle)

Given $\ell, \tau>0$ and a map $f$ as above, there exists $K=K(\ell, \tau, f)>1$ of the form

$$
K=\left(1+\frac{1}{\tau}\right)^{2} \exp C \ell,
$$

where $C$ is a constant depending only on $f$, with the following property. If $T$ is an interval such that $f^{m} \mid T$ is a diffeomorphism and if $\sum_{j=0}^{m-1}\left|f^{j}(T)\right| \leq \ell$, then for each interval $M \subseteq T$ for which the space of $f^{m}(M)$ inside $f^{m}(T)$ is at least $\tau$ and for all $x, y \in M$ we have

$$
\frac{1}{K} \leq\left|\frac{D f^{m}(x)}{D f^{m}(y)}\right| \leq K .
$$

Once again, see [16], p. 295, for a proof. Used in combination with Lemma 2.3, the Koebe distortion principle allows one to propagate space around under fairly general circumstances. 


\section{The real a-priori bounds}

In this section we establish real a-priori bounds for critical circle maps, obtaining as a corollary the fact that their renormalizations are pre-compact in the $C^{1}$ topology. The results are well-known, and the reader will not fail to notice the overlap with some of the material in [19] and [10].

Let $f: S^{1} \rightarrow S^{1}$ be a critical circle homeomorphism with critical point $c$. The iterates of $c$ are denoted by $c_{i}=f^{i}(c)$. Let $I_{n}$ be the interval with endpoints $c$ and $c_{q_{n}}$ that contains $c_{q_{n+2}}$, as defined in Sect. 2. For simplicity, we write $I_{n}^{j}=f^{j}\left(I_{n}\right)$ for all $j$ and $n$. The most basic combinatorial fact to be remembered here is that the collection of intervals

$$
\mathcal{P}_{n}=\left\{I_{n-1}, I_{n-1}^{1}, \ldots, I_{n-1}^{q_{n}-1}\right\} \cup\left\{I_{n}, I_{n}^{1}, \ldots, I_{n}^{q_{n-1}-1}\right\}
$$

constitutes a partition of $S^{1}$ modulo endpoints, called the dynamical partition of level $n$ associated to $f$. In order to get an actual partition we exclude from each interval in $\mathcal{P}_{n}$ its right endpoint, say, according to the standard choice of orientation of $S^{1}$. Let $\mathcal{P}_{n}(x)$ denote the atom of the partition $\mathcal{P}_{n}$ that contains $x$ (in particular, $\mathcal{P}_{n}(c)$ is either $I_{n}$ or $I_{n-1}$ according to the parity of $n$ ).

Theorem 3.1. (Real Bounds) Let $f \in \mathrm{Crit}^{r}\left(S^{1}\right)$ be a map with irrational rotation number. There exist constants $C_{0}>1$ and $0<\mu_{0}<\mu_{1}<1$ depending only on $f$ such that

(a) If I and $J$ are any two adjacent atoms of $\mathcal{P}_{n}$, then $C_{0}^{-1}|J|<|I|<C_{0}|J|$;

(b) For every $x \in S^{1}$, we have $\left|\mathcal{P}_{n}(x)\right|<\mu_{1}\left|\mathcal{P}_{n-1}(x)\right|$;

(c) If the rotation number of $f$ is of bounded type then $\left|\mathcal{P}_{n}(x)\right|>\mu_{0}^{n} / C_{0}$;

(d) If the rotation number of $f$ is of bounded type then $\left|\mathcal{P}_{n}(x)\right|>$ $\left|\mathcal{P}_{n-1}(x)\right| / C_{0}$

(e) If $0<i \leq j \leq q_{n}$ then the distortion of the restriction of $f^{j-i}$ to $I_{n-1}^{i}=f^{i}\left(I_{n-1}\right)$ is bounded by $C_{0}$.

In particular, the critical commuting pairs $\mathcal{R}^{n} f$ form a bounded sequence in the $C^{1}$ topology.

Later in this section we will see that the bounds in this theorem are eventually universal.

\subsection{Bounding space}

In what follows, two positive numbers $a$ and $b$ are said to be comparable modulo $f$, or simply comparable, if there exists a constant $C>1$, depending only on our map $f$, such that $C^{-1} b \leq a \leq C b$. This relation is denoted 
by $a \asymp b$. It is also convenient to write $a \preccurlyeq b$ to indicate that $a \leq C b$. Comparability modulo $f$ is reflexive and symmetric, but not transitive since the constants multiply. Hence, if $b_{1} \asymp b_{2} \asymp \cdots \asymp b_{N}$, we can only say that $b_{1} \asymp b_{N}$ if $N$ is bounded (by a constant depending only on $f$ ).

Lemma 3.2. For each $n \geq 0$ there exist $z_{1}, z_{2}, z_{3}, z_{4}, z_{5} \in S^{1}$ with $z_{j+1}=f^{q_{n}}\left(z_{j}\right)$ such that $\left|z_{1}-z_{2}\right| \asymp\left|z_{2}-z_{3}\right| \asymp\left|z_{3}-z_{4}\right| \asymp\left|z_{4}-z_{5}\right|$.

Proof. Let $z \in S^{1}$ be a point such that $\left|f^{q_{n}}(z)-z\right| \leq\left|f^{q_{n}}(x)-x\right|$ for all $x \in S^{1}$. From Koebe's principle applied successively to $f^{-q_{n}}, f^{-2 q_{n}}$ and $f^{-3 q_{n}}$, we have

$\left|z-f^{q_{n}}(z)\right| \succcurlyeq\left|f^{-q_{n}}(z)-z\right| \succcurlyeq\left|f^{-2 q_{n}}(z)-f^{-q_{n}}(z)\right| \succcurlyeq\left|f^{-3 q_{n}}(z)-f^{-2 q_{n}}(z)\right|$.

Moreover, by our choice of $z$ we have $\left|z-f^{q_{n}}(z)\right| \preccurlyeq\left|f^{-3 q_{n}}(z)-f^{-2 q_{n}}(z)\right|$. Therefore we can take $z_{5}=f^{q_{n}}(z), z_{4}=z, \ldots, z_{1}=f^{-3 q_{n}}(z)$ as the desired five points.

Lemma 3.3. Let $z_{1}, z_{2}, \ldots, z_{5}$ and $w_{0}, w_{1}, \ldots, w_{5}$ be points on the circle such that $z_{j+1}=f^{q_{n}}\left(z_{j}\right)$ and $w_{j+1}=f^{q_{n}}\left(w_{j}\right)$, and such that $w_{1}$ lies on the interval of endpoints $z_{1}$ and $z_{2}$ in the partition of $S^{1}$ determined by the $z_{i}$ 's. If $\left|z_{1}-z_{2}\right| \asymp\left|z_{2}-z_{3}\right| \asymp\left|z_{3}-z_{4}\right| \asymp\left|z_{4}-z_{5}\right|$, then

$$
\left|w_{0}-w_{1}\right| \succcurlyeq\left|w_{1}-w_{2}\right| \preccurlyeq\left|w_{2}-w_{3}\right| \text {. }
$$

Proof. Let $\ell=\min \left|z_{j}-z_{j+1}\right|$. If there is a $j$ with $1 \leq j \leq 3$ such that $\left|w_{j}-w_{j+1}\right| \leq \ell / 2$, then we must have $\left|w_{j-1}-w_{j}\right| \geq \ell / 2$ and $\left|w_{j+1}-w_{j+2}\right| \geq \ell / 2$ also. But then $\left[w_{j}, w_{j+1}\right]$ has space on both sides inside $\left[w_{j-1}, w_{j+2}\right]$. Applying $f^{-(j-1) q_{n}}$ to these points and using the Koebe principle, we get (1). If on the other hand there is no $j$ with that property, then $\left|w_{1}-w_{2}\right| \asymp\left|w_{2}-w_{3}\right| \asymp\left|w_{3}-w_{4}\right|$. Again, applying $f^{-q_{n}}$ and using Koebe we get (1).

Lemma 3.4. For all $n \geq 0$ and all $x \in S^{1}$, we have $\left|f^{q_{n}}(x)-x\right| \asymp$ $\left|x-f^{-q_{n}}(x)\right|$.

Proof. To show that $\left|x-f^{-q_{n}}(x)\right| \geq C^{-1}\left|f^{q_{n}}(x)-x\right|$, let $i \leq q_{n}$ be such that $f^{i}(x) \in\left[z_{1}, z_{2}\right]$, where $z_{1}, z_{2}, \ldots$ are the points given by Lemma 3.2. Then let $w_{0}=f^{i-q_{n}}(x), w_{1}=f^{i}(x)$, etc. We know from Lemma 3.3 that $\left|w_{0}-w_{1}\right| \succcurlyeq\left|w_{1}-w_{2}\right| \preccurlyeq\left|w_{2}-w_{3}\right|$. Applying $f^{-i}$ to these points and using the Koebe distortion principle, we find a definite space around $\left[x, f^{q_{n}}(x)\right]$ inside $\left[f^{-q_{n}}(x), f^{2 q_{n}}(x)\right]$. Therefore $\left|x-f^{-q_{n}}(x)\right| \succcurlyeq\left|f^{q_{n}}(x)-x\right|$. The proof of the opposite inequality is similar.

We arrive at the following fundamental fact first proved by Świagtek [18] and Herman [12]. 


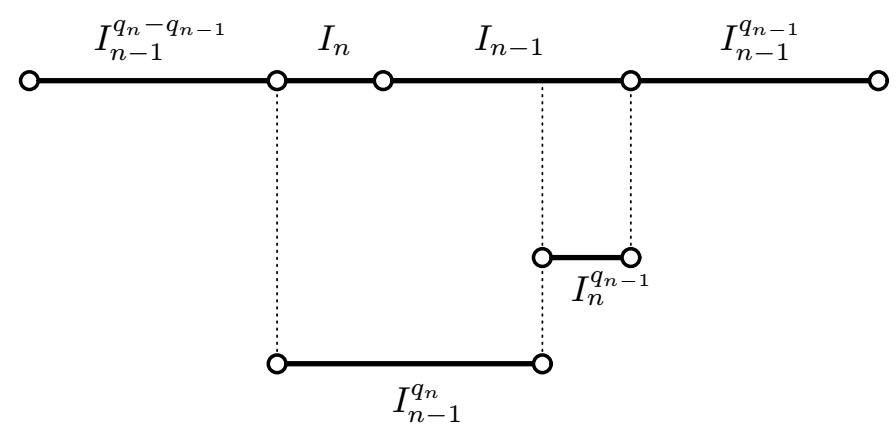

Fig. 2. These six intervals are pairwise comparable

Lemma 3.5. Any two adjacent intervals in the dynamical partition of level $n$ of $f$ are comparable.

Proof. First we prove that all intervals in Fig. 2 are pairwise comparable, through the following steps.

(a) From Lemma 3.4, we know that $\left|I_{n-1}\right| \asymp\left|I_{n-1}^{q_{n-1}}\right|$ and $\left|I_{n-1}^{q_{n}-q_{n-1}}\right| \asymp\left|I_{n-1}^{q_{n}}\right|$.

(b) Since the dynamical symmetric of $I_{n}$, namely the interval $I_{n}^{-q_{n}}$, is contained in $I_{n-1}$, we also have $\left|I_{n}\right| \preccurlyeq\left|I_{n-1}\right|$.

(c) Since the dynamical symmetric of $I_{n-1}$, namely $I_{n-1}^{-q_{n-1}}$ is contained in $I_{n} \cup I_{n-1}^{q_{n}-q_{n-1}}$, we have $\left|I_{n-1}\right| \preccurlyeq\left|I_{n-1}^{q_{n}-q_{n-1}}\right|$. Moreover, since $I_{n-1}^{q_{n}} \subseteq$ $I_{n} \cup I_{n-1}$, items $(a)$ and $(b)$ yield $\left|I_{n-1}^{q_{n}-q_{n-1}}\right| \preccurlyeq\left|I_{n-1}\right|$. Therefore $\left|I_{n-1}\right| \asymp$ $\left|I_{n-1}^{q_{n}-q_{n-1}}\right|$.

(d) Next, we claim that $\left|I_{n}\right| \asymp\left|I_{n}^{q_{n-1}}\right|$. To see why, consider the diffeomorphism

$$
f^{q_{n}-q_{n-1}}: I_{n-1} \cup I_{n-1}^{q_{n-1}} \rightarrow I_{n-1}^{q_{n}-q_{n-1}} \cup I_{n-1}^{q_{n}} .
$$

By the cross-ratio inequality (Lemma 2.3) applied to $M=I_{n}^{q_{n-1}}$ and $T=$ $I_{n-1} \cup I_{n-1}^{q_{n-1}}$, we have $\left|I_{n}^{q_{n-1}}\right| \preccurlyeq\left|I_{n}^{q_{n}}\right| \asymp\left|I_{n}\right|$. Conversely, considering the diffeomorphism

$$
f^{q_{n-1}}: I_{n-1}^{q_{n}-q_{n-1}} \cup I_{n} \rightarrow I_{n-1}^{q_{n}} \cup I_{n}^{q_{n-1}}
$$

and applying the cross-ratio inequality to $M=I_{n}^{q_{n}}$ and $T=I_{n-1}^{q_{n}-q_{n-1}} \cup$ $I_{n}$, we get

$$
\left|I_{n}\right| \asymp\left|I_{n}^{q_{n}}\right| \preccurlyeq\left|I_{n-1}^{q_{n}+q_{n-1}}\right| \asymp\left|I_{n}^{q_{n-1}}\right| .
$$

This proves our claim.

(e) Finally, we claim that $\left|I_{n-1}\right| \preccurlyeq\left|I_{n}\right|$, thereby reversing the inequality in $(b)$. It is here that we use the critical point in a crucial way. Let $\theta_{n}=\left|I_{n}\right| /\left|I_{n-1}\right|$; we already know that $\theta_{n} \preccurlyeq 1$. Look at the intervals $I_{n-1}^{1}, I_{n}^{1}$ and $I_{n-1}^{q_{n}-q_{n-1}+1}$, all near the critical value of $f$. By an argument 
similar to the one in $(c)$, we have $\left|I_{n-1}^{1}\right| \asymp\left|I_{n-1}^{q_{n}-q_{n-1}+1}\right|$. Moreover, $\left|I_{n}^{1}\right| \asymp \theta_{n}^{p}\left|I_{n-1}^{1}\right|$, where $p>1$ is the power-law of $f$ at the critical point. Hence these three intervals have a cross-ratio comparable to $\theta_{n}^{p}$. On the other hand the map $f^{q_{n-1}-1}$ carries them diffeomorphically onto $I_{n-1}^{q_{n-1}}, I_{n}^{q_{n-1}}$ and $I_{n-1}^{q_{n}}$, respectively, whose cross-ratio is comparable to $\left|I_{n}^{q_{n-1}}\right| /\left|I_{n-1}^{q_{n}}\right|$, which in turn is comparable to $\theta_{n}$. Applying the Koebe distortion principle, we see that $\theta_{n}^{p} \succcurlyeq \theta_{n}$, and so $\theta_{n} \succcurlyeq 1$ as claimed.

This proves that all six intervals in Fig. 2 are comparable. To derive the remaining comparability relations, propagate this information using Koebe's distortion principle.

Proof of Theorem 3.1. Part (a) is Lemma 3.5 above. The remaining statements are straightforward consequences of $(a)$.

\subsection{Beau property of renormalization}

The bounds obtained in the proof of Theorem 3.1 depended on $f$, more precisely on the space that each atom of $\mathcal{P}_{n}$ enjoys relative to its two neighbors in $\mathcal{P}_{n}$. We now concentrate in proving that such bounds eventually become universal. It suffices to prove that the space in question is eventually universal. Bounds of this type are called beau by Sullivan.

Lemma 3.6. There exists $n_{0}=n_{0}(f)$ such that for all $n \geq n_{0}$ the first return map $f_{n}: J_{n} \rightarrow J_{n}$ satisfies $S f_{n}(x)<0$ for all $x \in J_{n}$.

Proof. This is proved in Theorem A.4 of Appendix A.

Lemma 3.7. Given $\varepsilon>0$, there exists $n_{1}=n_{1}(f, \varepsilon)>n_{0}(f)$ such that the following holds for all $n \geq n_{1}$. Let $\Delta \in \mathcal{P}_{n}$, let $k \geq 1$ be an integer such that $f^{j}(\Delta)$ is contained in an element of $\mathcal{P}_{n}$ for all $1 \leq j \leq k$, and let $\Delta^{*}$ be the union of $\Delta$ with its left and right neighbors in $\mathcal{P}_{n}$. Then we have $f^{k} \mid \Delta^{*}=\phi_{1} \circ \phi_{2} \circ \phi_{3}$ where $\phi_{1}$ and $\phi_{3}$ are diffeomorphisms with distortion bounded by $1+\varepsilon$ and $\phi_{2}$ is either the identity or a map with negative Schwarzian derivative. In particular, if $\varepsilon$ is small enough and if $I_{n-1} \neq \Delta \neq I_{n}$, then the distortion of $f^{k} \mid \Delta$ is bounded from below by one-half.

Proof. Let $n_{1}>n_{0}$ be such that $\mu_{0}^{n_{1}-n_{0}} \ll \varepsilon$, where $\mu_{0}$ is the constant of Theorem 3.1. For $n \geq n_{1}, \Delta$ and $k$ as in the statement, let $J \in \mathcal{P}_{n_{0}}$ be such that $\Delta \subseteq J$, let $J^{*}$ be the union of $J$ with its two neighbors in $\mathcal{P}_{n_{0}}$, and note that the space of $\Delta^{*}$ inside $J^{*}$ is bounded from below by $C\left|J^{*}\right| /\left|\Delta^{*}\right|$, for some constant $C>0$. Let $m \geq 0$ be the smallest integer such that 
$f^{m}(J) \subseteq J_{n_{0}}$. Then for all $j \leq m$ the map $f^{j} \mid J^{*}$ is a diffeomorphism onto its image and, by Theorem 3.1 (b) and the Koebe distortion principle, its distortion on $\Delta^{*}$ is bounded by

$$
\left(1+C \frac{\left|\Delta^{*}\right|}{\left|J^{*}\right|}\right)^{2} \exp \left\{C \frac{\left|\Delta^{*}\right|}{\left|J^{*}\right|}\right\} \leq \exp \left\{C \mu_{0}^{n_{1}-n_{0}}\right\} \leq 1+\varepsilon .
$$

Now, there are two possibilities. The first is that $m \geq k$; in this case we can take $\phi_{1}=f^{k} \mid \Delta^{*}$ and $\phi_{2}=\phi_{3}=$ identity map. The second is that $m<k$. In this case we consider the first return map $f_{n_{0}}: J_{n_{0}} \rightarrow J_{n_{0}}$ and let $\ell \geq 0$ be the largest such that

$$
f^{k}=f^{k_{1}} \circ f_{n_{0}}^{\ell} \circ f^{k_{3}},
$$

where $k_{1} \geq 0$ and $k_{3} \geq 0$. We then take $\phi_{1}=f^{k_{1}-1}, \phi_{2}=f \circ f_{n_{0}}^{\ell}$ and $\phi_{3}=f^{k_{3}} \mid \Delta^{*}$ (if $k_{1}=0$ we take instead $\phi_{2}=f_{n_{0}}^{\ell}$ and $\phi_{1}=$ identity). By Lemma 3.6, $S \phi_{2}<0$, and by the above remarks the distortions of both $\phi_{1}$ and $\phi_{3}$ are bounded by $1+\varepsilon$ in the appropriate domains.

Proposition 3.8. All bounds in Theorem 3.1 are beau. In other words, there exist universal constants $K_{0}>0$ and $0<\lambda_{0}<\lambda_{1}<1$ and some $\bar{n}=\bar{n}(f)>0$ such that for all $n \geq \bar{n}$ the constants $C_{0}, \mu_{0}$ and $\mu_{1}$ in Theorem 3.1 can be replaced by $K_{0}, \lambda_{0}$ and $\lambda_{1}$, respectively.

Proof. This is straightforward from Lemma 3.7.

Remark. From now on, whenever we say that a constant "depends only on the real bounds", we mean that the said constant is a universal function of constants $K_{0}, \lambda_{0}$ and $\lambda_{1}$ given by this proposition.

\section{How smooth is the conjugacy?}

Now we turn to the first main result in this paper. The theorem states that if the successive renormalizations of two critical circle maps with the same rotation number $\rho$ converge together at an exponential rate, then such maps are $C^{1+\alpha}$ conjugate for some $\alpha>0$, provided $\rho$ belongs to a certain fullmeasure set $\mathbb{A}$ of rotation numbers (defined in $\S 4.4$ ). First, in order to get bounds that do not depend on the maximum of the partial quotients of the rotation number, we need to perform some "saddle-node" estimates and constructions. 


\subsection{Saddle-node geometry}

Let $a$ be a positive integer and $\Delta_{1}, \Delta_{2}, \ldots, \Delta_{a+1}$ be consecutive intervals on the line or circle. By an almost parabolic map of length $a$ and fundamental domains $\Delta_{j}, 1 \leq j \leq a$, we mean a negative-Schwarzian diffeomorphism

$$
f: \Delta_{1} \cup \Delta_{2} \cup \cdots \cup \Delta_{a} \rightarrow \Delta_{2} \cup \Delta_{3} \cup \cdots \cup \Delta_{a+1}
$$

such that $f\left(\Delta_{j}\right)=\Delta_{j+1}$.

The basic geometric estimate on almost parabolic maps is due to J.-C. Yoccoz.

Yoccoz's Lemma. Let $f: I \rightarrow J$ be an almost parabolic map of length a and fundamental domains $\Delta_{j}, 1 \leq j \leq a$. If $\left|\Delta_{1}\right| \geq \sigma|I|$ and $\left|\Delta_{a}\right| \geq \sigma|I|$, then

$$
\frac{1}{C_{\sigma}} \frac{|I|}{\min \{j, a-j\}^{2}} \leq\left|\Delta_{j}\right| \leq C_{\sigma} \frac{|I|}{\min \{j, a-j\}^{2}},
$$

where $C_{\sigma}>1$ depends only on $\sigma$.

For a proof, see Appendix B. We will use Yoccoz's estimates to compare two almost parabolic maps.

Proposition 4.1. Let $f$ and $g$ be two almost parabolic maps with the same length a defined on the same interval. Then for all $x \in \Delta_{1}(f) \cap \Delta_{1}(g)$ and all $0 \leq k \leq a / 2$ we have

$$
\left|f^{k}(x)-g^{k}(x)\right| \leq C\|f-g\|_{0} k^{3} .
$$

Proof. First note, using the mean-value theorem, that

$$
\begin{aligned}
\left|f^{k}(x)-g^{k}(x)\right| & =\left|\sum_{j=0}^{k-1}\left(f^{k-j-1}\left(f\left(g^{j}(x)\right)\right)-f^{k-j-1}\left(g^{j+1}(x)\right)\right)\right| \\
& \leq \sum_{j=0}^{k-1}\left|D f^{k-j-1}\left(\xi_{j}\right)\right|\left|f\left(g^{j}(x)\right)-g\left(g^{j}(x)\right)\right|,
\end{aligned}
$$

where $\xi_{j}$ lies between $f\left(g^{j}(x)\right)$ and $g^{j+1}(x)$. Hence we have

$$
\left|f^{k}(x)-g^{k}(x)\right| \leq\|f-g\|_{0} \sum_{j=0}^{k-1}\left|D f^{k-j-1}\left(\xi_{j}\right)\right| .
$$

Let us estimate each summand in the right-hand side of (3). Let $m=$ $m(j)$ be such that $\xi_{j} \in \Delta_{j+m}(f)$, and assume also that $j+m \leq a / 2$. This last condition is always satisfied if the central fundamental domain of $g$ lies 
to the left of the central fundamental domain of $f$ (if this is not the case, then reverse the roles of $f$ and $g$ in (3) and throughout). Using Yoccoz's Lemma, we see that

$$
\left|D f^{k-j-1}\left(\xi_{j}\right)\right| \asymp \frac{(j+m)^{2}}{(a-k-m+1)^{2}} \leq\left(\frac{j+m}{j+1}\right)^{2} .
$$

Hence, it suffices to estimate $m$ as a function of $j$. For this purpose, let $n=n(j)$ be such that $g^{j+1}(x) \in\left[f^{j+n-1}(x), f^{j+n}(x)\right]$. We claim that $m \leq n+1$. There are two possibilities. The first is that $f\left(g^{j}(x)\right) \geq g^{j+1}(x)$ : in this case we see easily that

$$
\xi_{j} \in\left[g^{j+1}(x), f\left(g^{j}(x)\right)\right] \subseteq\left[f^{j+n-1}(x), f^{j+n+1}(x)\right]
$$

and so $m \leq n+1$. The second is that $f\left(g^{j}(x)\right)<g^{j+1}(x)$. In this case we have $\xi_{j}<g^{j+1}(x)<f^{j+n}(x) \in \Delta_{j+n+1}(f)$, so once again $m \leq n+1$. This proves our claim.

So now we must bound $n$ as a function of $j$. Again, there are two cases to consider.

(a) We have $\left[g^{j+1}(x), g^{j+2}(x)\right] \subseteq\left[f^{j+n-1}(x), f^{j+n}(x)\right]$. In this case (Fig. 3a) Yoccoz's Lemma gives us

$$
\frac{1}{j^{2}} \leq \frac{C}{(j+n)^{2}},
$$

which implies $n \leq C j$.

(a)

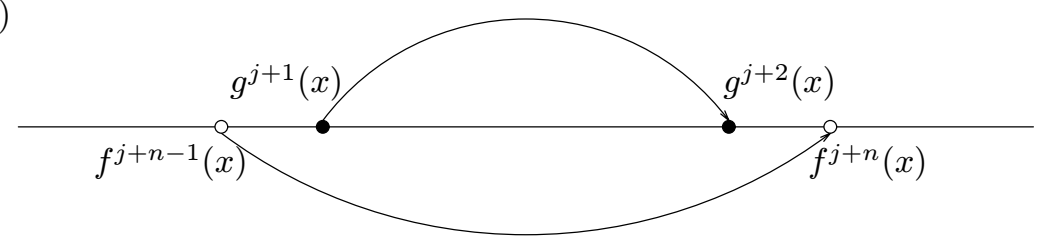

(b)

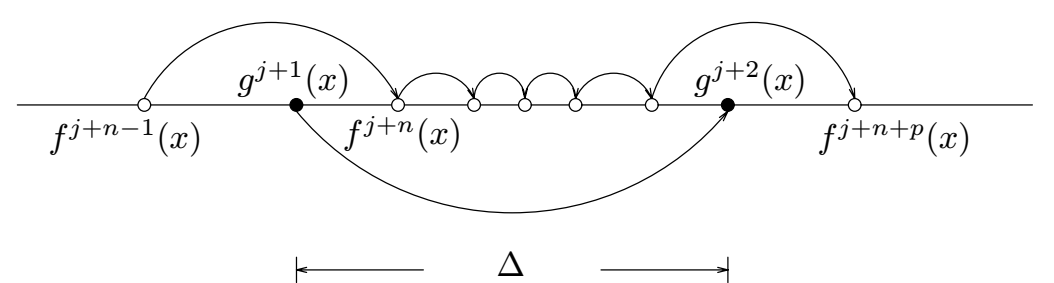

Fig. 3. Bounding $n$ as a function of $j$ 
(b) We have $g^{j+2}(x)>f^{j+n}(x)$. In this case (Fig. $\left.3 b\right), f^{j+n}(x)$ is the first point in the $f$-orbit of $x$ that lands inside the interval $\Delta=\left[g^{j+1}(x), g^{j+2}(x)\right]$. Let $p$ be such that $f^{j+n+i}(x) \in \Delta$ for $i=0,1, \ldots, p-1$ but $f^{j+n+p}(x) \notin \Delta$. Then we have $\Delta \subseteq$ $\left[f^{j+n-1}(x), f^{j+n+p}(x)\right]$, and this time Yoccoz's Lemma gives us

$$
\frac{1}{j^{2}} \leq C\left(\frac{1}{(j+n)^{2}}+\frac{1}{(j+n+1)^{2}}+\cdots+\frac{1}{(j+n+p)^{2}}\right) \leq \frac{C}{j+n} .
$$

Therefore $n \leq C j^{2}$ in this case.

In either case we see that $m \leq C j^{2}$. Carrying this information back to (4), we deduce that

$$
\left|D f^{k-j-1}\left(\xi_{j}\right)\right| \leq C j^{2} .
$$

Substituting (5) into (3), we arrive at (2), and the proof is complete.

\subsection{A criterion for smoothness}

One key ingredient in the proof of our First Main Theorem is a slight extension of a result originally due to Carleson [2], namely Proposition 4.3 below. To formulate it, we need an auxiliary definition.

Definition. A fine grid is a sequence $\left\{\mathcal{Q}_{n}\right\}_{n \geq 0}$ of finite partitions of $S^{1}$ which satisfies

(a) Each $\mathcal{Q}_{n+1}$ is a strict refinement of $\mathcal{Q}_{n}$;

(b) There exists $a>0$ such that each $I \in \mathcal{Q}_{n}$ is the disjoint union of at most $a$ atoms of $\mathcal{Q}_{n+1}$;

(c) There exists $c>0$ such that $c^{-1}|I| \leq|J| \leq c|I|$ for each pair of adjacent atoms $I, J \in \mathcal{Q}_{n}$.

For example, the dynamical partitions $\left\{\mathcal{P}_{n}\right\}$ of a critical circle map with rotation number of bounded type always form a fine grid, by Theorem 3.1. We note the following easy lemma concerning a fine grid $\left\{\mathcal{Q}_{n}\right\}$.

Lemma 4.2. If $I \in \mathcal{Q}_{n}, J \in \mathcal{Q}_{n+1}$ and $J \subseteq I$, then $\left(1+c^{-1}\right)|J| \leq|I| \leq$ $a c^{a}|J|$. In particular, there exist $C_{0}>1$ and $0<\lambda_{0}<\lambda_{1}<1$ such that $C_{0}^{-1} \lambda_{0}^{n} \leq|I| \leq C_{0} \lambda_{1}^{n}$, for all $I \in \mathcal{Q}_{n}$.

The constants $a, c, C_{0}, \lambda_{0}, \lambda_{1}$, are the fine constants of $\left\{\mathcal{Q}_{n}\right\}$.

Proposition 4.3. Let $h: S^{1} \rightarrow S^{1}$ be a homeomorphism and let $\left\{\mathcal{Q}_{n}\right\}_{n \geq 0}$ be a fine grid. 
(a) If there exists $C>0$ such that

$$
\left|\frac{|I|}{|J|}-\frac{|h(I)|}{|h(J)|}\right| \leq C,
$$

for each pair of adjacent atoms $I, J \in \mathcal{Q}_{n}$, for all $n \geq 0$, then $h$ is quasisymmetric.

(b) If there exist constants $C>0$ and $0<\lambda<1$ such that

$$
\left|\frac{|I|}{|J|}-\frac{|h(I)|}{|h(J)|}\right| \leq C \lambda^{n},
$$

for each pair of adjacent atoms $I, J \in \mathcal{Q}_{n}$, for all $n \geq 0$, then $h$ is a $C^{1+\alpha}$-diffeomorphism for some $\alpha>0$.

The proof of Proposition 4.3 will depend on the following fact from elementary real analysis. If $\phi$ is a real-valued function in an interval or oriented arc on the circle, let $D^{+} \phi(x)=\lim _{t \downarrow 0}(\phi(x+t)-\phi(t)) / t$ be the right derivative of $\phi$ at $x$, if the limit exists.

Lemma 4.4. Let $\phi_{n}:[0,1] \rightarrow \mathbb{R}$ be a sequence of continuous, right differentiable mappings such that $D^{+} \phi_{n}$ converges uniformly to an $\alpha$ Hölder continuous function $\varphi:[0,1] \rightarrow \mathbb{R}$, and such that each $D^{+} \phi_{n}$ is Riemann-integrable. If $\phi_{n}$ converges uniformly to $\phi$, then $\phi$ is $C^{1+\alpha}$ and $D \phi=\varphi$.

Proof of Proposition 4.3. We will prove (b) only, the proof of (a) being somewhat easier. Let $\phi_{n}$ be the piecewise affine $C^{0}$-approximations to $h$ determined by the vertices of $\mathcal{Q}_{n}$. Then $\phi_{n}$ is differentiable on the right, and $D^{+} \phi_{n}$ is a step function. First we show that $\left\{D^{+} \phi_{n}\right\}_{n>0}$ is a uniform Cauchy sequence, and then that the limit is Hölder continuous. Take an atom $I$ of $\mathcal{Q}_{n}$, and consider the decomposition

$$
I=J_{1} \cup J_{2} \cup \cdots \cup J_{p},
$$

with $J_{k} \in \mathcal{Q}_{n+1}$ consecutive and pairwise disjoint and $p \leq a$. Then $D^{+} \phi_{n}$ is constant on $I$ and $D^{+} \phi_{n+1}$ is constant on each $J_{k}$, say

$$
\left\{\begin{array}{ll}
D^{+} \phi_{n}(t)=\sigma=\frac{\left|\phi_{n}(I)\right|}{|I|} & (t \in I) \\
D^{+} \phi_{n+1}(t)=\sigma_{k}=\frac{\left|\phi_{n+1}\left(J_{k}\right)\right|}{\left|J_{k}\right|} & \left(t \in J_{k}\right)
\end{array} .\right.
$$

Thus, we have

$$
\sigma|I|=\sum_{k=1}^{p} \sigma_{k}\left|J_{k}\right|
$$


and in particular $\sigma^{\prime}=\min \sigma_{k} \leq \sigma \leq \max \sigma_{k}=\sigma^{\prime \prime}$. Also, $\sigma^{\prime} / \sigma^{\prime \prime} \leq \sigma / \sigma_{k} \leq$ $\sigma^{\prime \prime} / \sigma^{\prime}$ for all $k$. Since by assumption $\left|1-\left(\sigma_{k+1} / \sigma_{k}\right)\right| \leq C \lambda^{n+1}$, an easy telescoping trick gives us

$$
\frac{\sigma^{\prime \prime}}{\sigma^{\prime}} \leq\left(1+C \lambda^{n+1}\right)^{a} \leq 1+C \lambda^{n+1} .
$$

A similar lower bound holds true for $\sigma^{\prime} / \sigma^{\prime \prime}$. Therefore we have

$$
1-C \lambda^{n} \leq \frac{\sigma}{\sigma_{k}} \leq 1+C \lambda^{n},
$$

for all $k=1,2, \ldots p$. This shows that the sequence $\left\{D^{+} \phi_{n}\right\}_{n>0}$ is uniformly bounded, and moreover that for all $m \geq n \geq 0$ and all $t \in S^{1}$, we have

$$
\left|D^{+} \phi_{m}(t)-D^{+} \phi_{n}(t)\right| \leq C \sum_{j=n}^{m-1} \lambda^{j}<\frac{C}{1-\lambda} \lambda^{n} .
$$

Hence $\left\{D^{+} \phi_{n}\right\}_{n \geq 0}$ is a uniform Cauchy sequence as claimed. Let $\varphi=$ $\lim D^{+} \phi_{n}$, and let $\alpha>0$ be such that $\lambda_{0}^{\alpha}=\lambda$. We prove $\varphi$ is $\alpha$-Hölder as follows. It suffices to consider points $x, y \in S^{1}$ whose distance is smaller than $\inf _{I \in \mathcal{Q}_{0}}|I|$. Take the smallest $n$ such that $x$ and $y$ belong to distinct elements of $\mathcal{Q}_{n}$. Then either $n=0$ or $x$ and $y$ lie in a common element of $\mathcal{Q}_{n-1}$. Either way we have by (7)

$$
\left|D^{+} \phi_{n}(x)-D^{+} \phi_{n}(y)\right| \leq C \lambda^{n} .
$$

Combining (8) and (9), we deduce that

$$
\begin{aligned}
|\varphi(x)-\varphi(y)| \leq & \left|\varphi(x)-D^{+} \phi_{n}(x)\right|+\left|D^{+} \phi_{n}(x)-D^{+} \phi_{n}(y)\right| \\
& +\left|D^{+} \phi_{n}(y)-\varphi(y)\right| \\
\leq & \frac{C}{1-\lambda} \lambda^{n}+C \lambda^{n}+\frac{C}{1-\lambda} \lambda^{n} \leq C \lambda_{0}^{n \alpha} \\
\leq & C|x-y|^{\alpha},
\end{aligned}
$$

and so $\varphi$ is $\alpha$-Hölder as claimed.

Remark. In the language of conditional expectations, the sequence $\left\{D^{+} \phi_{n}\right\}_{n \geq 0}$ satisfies $\mathbb{E}\left(D^{+} \phi_{n} \mid \mathcal{B}_{n}\right)=D^{+} \phi_{n+1}$, where $\mathcal{B}_{n}$ is the $\sigma$-algebra generated by $\mathcal{Q}_{n}$, and therefore constitutes a martingale. Thus, the existence of a pointwise a.e. limit $\varphi$, merely as an integrable function, is a special case of J. Doob's martingale convergence theorem, see [1], p. 490. 


\subsection{A suitable fine grid}

The dynamical partitions $\mathcal{P}_{n}$ of a critical circle map $f$ do not determine a fine grid, unless the rotation number of $f$ is of bounded type. We will however use these dynamical partitions to build a fine grid $\left\{\mathcal{Q}_{n}\right\}$ for our map $f$. The construction requires some preliminary definitions.

An element $I \in \mathcal{P}_{n}$ is a saddle-node atom if it is the disjoint union of some number $a \geq 1000$ of atoms of $\mathcal{P}_{n+1}$.

Given two atoms $\mathcal{P}_{n+1} \ni J \subseteq I \in \mathcal{P}_{n}$, the order of $J$ inside $I$ is one plus the smallest number of atoms of $\mathcal{P}_{n+1}$ on the right and left components of $I \backslash J$.

Note that inside a saddle-node atom $I \in \mathcal{P}_{n}$ there are exactly two atoms of $\mathcal{P}_{n+1}$ of order $k$ for each $k \leq a / 2$. Let $N \geq 0$ be largest with the property that $2^{N+1}<a / 2$. For each $0 \leq i \leq N$, we define $M_{i}$, the $i$-th central interval of $I$, to be the convex-hull $\left[J, J^{*}\right] \subseteq I$ of the union of both atoms $J, J^{*}$ of order $2^{i}$. Note that these central intervals are nested (see Fig. 4). The left and right components of $M_{i} \backslash M_{i+1}$, respectively $L_{i}$ and $R_{i}$, are the lateral intervals of $I$. The central interval $M_{N}$ is also called the final interval of $I$. The lateral intervals together with the final interval form a special partition of $I$, the balanced partition of $I$.

Remark. It follows from Yoccoz's lemma that $\left|L_{i}\right| \asymp\left|M_{i+1}\right| \asymp\left|R_{i}\right|$ for all $i$.

Now we define an auxiliary partition $\widetilde{\mathcal{P}}_{n}$, for each $n \geq 1$. The atoms of $\widetilde{\mathcal{P}_{n}}$ are all atoms of $\mathcal{P}_{n}$ which are not saddle-node, together with the atoms

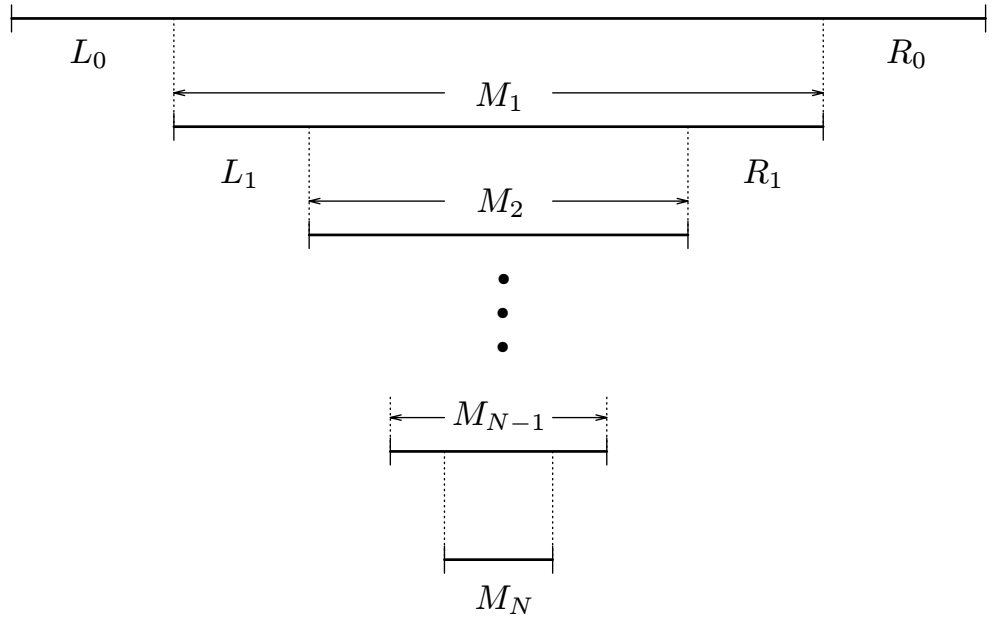

Fig. 4. Central and lateral intervals of a saddle-node atom 
of the balanced partitions of all saddle-node atoms of $\mathcal{P}_{n}$. The partition $\mathcal{Q}_{n}$ that we want is constructed from $\widetilde{\mathcal{P}}_{n}$ and $\mathcal{P}_{n}$ as follows.

Proposition 4.5. There exists a fine grid $\left\{\mathcal{Q}_{n}\right\}$ in $S^{1}$ with the following properties.

(a) Every atom of $\mathcal{Q}_{n}$ is the union of at most 3 atoms of $\mathcal{Q}_{n+1}$.

(b) Every atom $\Delta$ of $\mathcal{Q}_{n}$ is a union of atoms of $\mathcal{P}_{m}$ for some $m \leq n$, and there are four possibilities:

$\left(b_{1}\right) \Delta$ is a single atom of $\mathcal{P}_{m}$;

$\left(b_{2}\right) \Delta$ is a central interval of $\widetilde{\mathcal{P}}_{m}$;

$\left(b_{3}\right) \Delta$ is the union of at least two atoms of $\mathcal{P}_{m+1}$ contained in a single atom of $\widetilde{\mathcal{P}}_{m}$.

$\left(b_{4}\right) \Delta$ is a union of intervals which are simultaneously atoms of $\mathcal{P}_{m}$ and $\widetilde{\mathcal{P}}_{m}$.

Proof. The proof is by induction on $n$. The first partition $\mathcal{Q}_{1}$ consists of all atoms of $\mathcal{P}_{1}$ which are not saddle-node atoms together with the intervals $L_{0}, M_{1}$ and $R_{0}$ of each saddle-node interval $I \in \mathcal{P}_{1}\left(I=L_{0} \cup M_{1} \cup R_{0}\right)$. It is clear that each atom of $\mathcal{Q}_{1}$ falls within one of the categories $\left(b_{1}\right)-\left(b_{4}\right)$ above.

Assuming $\mathcal{Q}_{n}$ defined, define $\mathcal{Q}_{n+1}$ as follows. Take an atom $I \in \mathcal{Q}_{n}$ and consider the four cases below.

(1) If $I$ is a single atom of $\mathcal{P}_{m}$ then one of two things can happen:

(i) $I$ is a saddle-node atom: In this case write $I=L_{0} \cup M_{1} \cup R_{0}$ as above and take $L_{0}, R_{0}$ and $M_{1}$ as atoms of $\mathcal{Q}_{n+1}$. Note that the lateral intervals $L_{0}$ and $R_{0}$ are atoms of type $\left(b_{1}\right)$, while the central interval $M_{1}$ is of type $\left(b_{2}\right)$.

(ii) $I$ is not a saddle-node atom: In this case write $I=L \cup M \cup R$ where $L$ and $R$ are the atoms of $\mathcal{P}_{m+1}$ adjacent to the endpoints of $I$ and $M$ is the union of the other atoms of $\mathcal{P}_{m+1}$ inside $I$. Add these three intervals to $\mathcal{Q}_{n+1}$, noting that $L$ and $R$ are of type $\left(b_{1}\right)$, while $M$ is of type $\left(b_{4}\right)$.

(2) If $I$ is a central interval of $\widetilde{\mathcal{P}}_{m}$ which is not the final interval, consider the next central interval of $\widetilde{\mathcal{P}}_{m}$ inside $I$, say $M$, and the two corresponding lateral intervals $L$ and $R$ such that $I=L \cup M \cup R$, and declare $L, R$ and $M$ members of $\mathcal{Q}_{n+1}$. Note that $L$ and $R$ are of type $\left(b_{3}\right)$, while $M$ is of type $\left(b_{2}\right)$.

(3) If $I$ is a union of $p \geq 2$ consecutive atoms $\Delta_{1}, \ldots, \Delta_{p}$ of $\widetilde{\mathcal{P}}_{m+1}$ inside a single atom of $\mathcal{P}_{m}$, divide it up into three approximately equal parts. More precisely, write $p=3 q+r$ and, when $r=0$ or 1 , consider 
$I=L \cup M \cup R$ where

$$
L=\bigcup_{j=1}^{q} \Delta_{j}, M=\bigcup_{j=q+1}^{p-q} \Delta_{j}, R=\bigcup_{j=p-q+1}^{p} \Delta_{j} .
$$

When $r=2$, consider $I=L \cup M \cup R$ where

$$
L=\bigcup_{j=1}^{q+1} \Delta_{j}, M=\bigcup_{j=q+2}^{p-q-1} \Delta_{j}, R=\bigcup_{j=p-q}^{p} \Delta_{j} .
$$

Note that $M$ is empty when $p=2$. In any case, we obtain two or three new atoms of $\mathcal{Q}_{n+1}$ which are either single atoms of $\mathcal{P}_{m+1}$, and therefore of type $\left(b_{1}\right)$, or once again intervals of type $\left(b_{3}\right)$.

(4) If $I$ is a union of intervals which are simultaneously atoms of $\mathcal{P}_{m}$ and $\widetilde{\mathcal{P}}_{m}$, divide it up exactly as in (3), obtaining either two or three new atoms of $\mathcal{Q}_{n+1}$ which are either single atoms of $\mathcal{P}_{m}$, and therefore of type $\left(b_{1}\right)$, or once again intervals of type $\left(b_{4}\right)$.

This completes the induction. That $\left\{\mathcal{Q}_{n}\right\}_{n \geq 0}$ constitutes a fine grid follows easily from the real bounds and the remark preceding this proposition.

An immediate consequence of the mere existence of such a fine grid is the fact that any two critical circle maps with the same rotation number are quasisymmetrically conjugate.

Corollary 4.6. Let $f$ and $g$ be critical circle maps with the same irrational rotation number, and let $h$ be the conjugacy between $f$ and $g$ that maps the critical point of $f$ to the critical point of $g$. Then $h$ is quasisymmetric.

Proof. Apply Proposition 4.3 (a) to the fine grid constructed above.

\subsection{Proof of the First Main Theorem}

We will proceed according to the following strategy. Given two critical circle maps $f$ and $g$ with the same rotation number, consider the special partitions $\mathcal{Q}_{n}=\mathcal{Q}_{n}(f)$ and $\widetilde{\mathcal{Q}}_{n}=\mathcal{Q}_{n}(g)$ given by Proposition 4.5. The conjugacy $h$ between $f$ and $g$ is an isomorphism between the corresponding fine grids. We want to show that the coherence property (6) holds for $h$ and $\left\{\mathcal{Q}_{n}\right\}$. The stated $C^{1+\alpha}$ smoothness of $h$ will then follow from Proposition 4.3.

To achieve our goal, we need to impose certain conditions on the common rotation number of $f$ and $g$. Let us consider the set $\mathbb{A} \subseteq[0,1]$ of rotation numbers whose partial quotients $\left(a_{n}\right)$ satisfy

$$
\lim \sup _{n \rightarrow \infty} \frac{1}{n} \sum_{j=1}^{n} \log a_{j}<\infty
$$


as well as

$$
\lim _{n \rightarrow \infty} \frac{1}{n} \log a_{n}=0
$$

and which also satisfy

$$
\frac{1}{n} \sum_{j=k+1}^{k+n} \log a_{j} \leq \omega\left(\frac{n}{k}\right),
$$

for all $0<n \leq k$, where $\omega(t)$ is a positive function (that depends on the rotation number) defined for $t>0$ such that $t \omega(t) \rightarrow 0$ as $t \rightarrow 0$. This is the class of rotation numbers for which we shall prove our theorem. Note that all numbers of bounded type satisfy (10), (11) and (12). The number whose partial quotients are given by $a_{n}=k$ if $n=2^{k}$ with $k \geq 1$ and $a_{n}=1$ otherwise is an explicit element of $\mathbb{A}$ that is not of bounded type. This number satisfies (12) with $\omega(t)=1 / \sqrt{t}$. It is a well-known consequence of the fact that the Gauss map preserves a measure absolutely continuous with respect to Lebesgue measure (the Gauss measure) and Birkhoff's ergodic theorem that both (10) and (11) hold Lebesgue almost everywhere (see [3], page 175). Condition (12) also holds for Lebesgue almost all numbers if we take $\omega(t)=C(1-\log t)$, where $C>0$ depends on the number (this last fact was communicated to us by C.G. Moreira - for a proof, see Appendix C). Therefore our set $\mathbb{A}$ has full Lebesgue measure in $[0,1]$.

In what follows we use the notation $x_{n}=x_{n}(f)=f^{q_{n}}(c)$. We also assume that the critical point $c$ is the same for both maps. If the renormalizations $\boldsymbol{f}_{n}$ and $\boldsymbol{g}_{n}$ converge together exponentially fast then $\left|x_{n}(f)-c\right| /\left|x_{n}(g)-c\right|$ converges to a limit exponentially fast also. More precisely, we have the following lemma.

Lemma 4.7. If $\left\|\boldsymbol{f}_{n}-\boldsymbol{g}_{n}\right\|_{0} \leq C \mu^{k}$ for some $0<\mu<1$ and all $n \geq 0$, then the ratio $\left|x_{n}(f)-c\right| /\left|x_{n}(g)-c\right|$ converges to a limit exponentially fast. Moreover, for all $m, k \geq 1$ we have

$$
\left|\frac{\left|I_{m}(f)\right|}{\left|I_{k}(f)\right|}-\frac{\left|I_{m}(g)\right|}{\left|I_{k}(g)\right|}\right| \leq C \mu^{\min \{m, k\}} \frac{\left|I_{m}(f)\right|}{\left|I_{k}(f)\right|} .
$$

Proof. The hypothesis tells us that

$$
\left|\frac{\left|I_{n+1}(f)\right|}{\left|I_{n}(f)\right|}-\frac{\left|I_{n+1}(g)\right|}{\left|I_{n}(g)\right|}\right| \leq C_{1} \mu^{n}
$$

for all $n \geq 1$. Writing $\alpha_{n}=\left|x_{n}(f)-c\right| /\left|x_{n}(g)-c\right|=\left|I_{n}(f)\right| /\left|I_{n}(g)\right|$, and taking into account that $C_{2}^{-1}\left|I_{n}(g)\right| \leq\left|I_{n+1}(g)\right| \leq C_{2}\left|I_{n}(g)\right|$ by the real bounds (for some $C_{2}>1$ ), we see that the above inequality is equivalent to

$$
\left|\frac{\alpha_{n+1}}{\alpha_{n}}-1\right| \leq C_{3} \mu^{n}
$$


This is the same as $\alpha_{n+1}=\left(1+\varepsilon_{n}\right) \alpha_{n}$ where $\left|\varepsilon_{n}\right| \leq C_{3} \mu^{n}$. Therefore $\alpha_{n}=\alpha_{1} \prod_{j=1}^{n-1}\left(1+\varepsilon_{j}\right)$, and this shows that $\lim \alpha_{n}$ exists. Finally, note that if $m>k \geq 1$ then

$$
\begin{aligned}
\left|\frac{\alpha_{m}}{\alpha_{k}}-1\right| & \leq\left|\prod_{j=k}^{m-1}\left(1+\varepsilon_{j}\right)-1\right| \\
& \leq C_{4} \sum_{j=k}^{m-1} \varepsilon_{j}<C_{5} \mu^{k},
\end{aligned}
$$

and similarly for $\left|1-\alpha_{k} / \alpha_{m}\right|$, and these facts clearly imply (13).

Remark. Having established this lemma, we may assume, after conjugating one of the maps (say $g$ ) by a suitable smooth diffeomorphism, that the limit of the ratios $\left|I_{n}(f)\right| /\left|I_{n}(g)\right|$ is in fact equal to one. This will be our standing hypothesis from now on (used at the end of the proof of Lemma 4.10 below).

Definition. Let $f_{m}: J_{m}(f) \rightarrow J_{m}(f)$ be the $m$-th first return map of $f$ and let $k \neq 0$ be an integer such that $|k| \leq\left\lceil a_{m} / 2\right\rceil$ (where $\lceil x\rceil$ denotes the smallest integer $\geq x$ ). The restricted domain of $f_{m}^{k}$, denoted $D_{m, k}$, is defined as follows.

$$
D_{m, k}= \begin{cases}I_{m} \cup\left[f_{m}^{\left\lceil\frac{a_{m}}{2}\right\rceil-k}\left(x_{m-1}\right), x_{m-1}\right], & \text { when } k>0 \\ {\left[f_{m}\left(x_{m+1}\right), f_{m}^{\left\lceil\frac{a_{m}}{2}\right\rceil-k}\left(x_{m+1}\right)\right],} & \text { when } k \leq-1\end{cases}
$$

In less precise terms, the restricted domain $D_{m, k}$ is the the set of points in $J_{m}$ which can be iterated $k$ times by $f_{m}$ without ever going across the central fundamental domain of $f_{m}$ in $J_{m}(f) \backslash J_{m+1}(f)$.

Lemma 4.8. For all $x \in D_{m, k}$ we have $\left|D f_{m}^{k}(x)\right| \leq K$, where $K \geq 1$ depends only on the real bounds.

Proof. Use Theorem 3.1 and Yoccoz's Lemma.

Lemma 4.9. Let $v$ be a vertex of $\mathcal{P}_{k+p}(f)$ such that $v \in J_{k}(f)$. Then there exist $k \leq m \leq k+p$ and $1 \leq N \leq p$ such that $v$ can be represented in the form

$$
v=\phi_{1} \circ \phi_{2} \circ \cdots \circ \phi_{N}\left(x_{m}\right)
$$

where $\phi_{j}=f_{m_{j}}^{k_{j}}$ for some $k \leq m_{j} \leq k+p$ and $\left|k_{j}\right| \leq\left\lceil a_{m_{j}} / 2\right\rceil$, and where the point $\phi_{j+1} \circ \cdots \circ \phi_{N}\left(x_{m}\right)$ belongs to the restricted domain of $\phi_{j}$ for each $j$. 
Proof. For simplicity of notation, we write $J_{i}=J_{i}(f)$ in this proof. Let $k \leq m_{1} \leq k+p$ be largest with the property that $v \in J_{m_{1}} \backslash J_{m_{1}+1}$, and let $0<i \leq a_{m_{1}}$ be such that $f_{m_{1}}^{i}(v) \in J_{m_{1}+1}$. If $i \leq\left\lceil a_{m_{1}} / 2\right\rceil$ then let $k_{1}=-i$; otherwise let $k_{1}=a_{m_{1}}-i$. We get $\phi_{1}=f_{m_{1}}^{k_{1}}$ and a new vertex $v_{1}=f_{m_{1}}^{-k_{1}}(v) \in J_{m_{1}+1}$. If $v_{1} \in J_{k+p}$ then $v_{1}=f_{k+p}\left(x_{k+p-1}\right)$ necessarily, and we can stop. On the other hand, if $v_{1} \notin J_{k+p}$, then once again there exists $m_{2}$ in the range $m_{1}<m_{2}<k+p$ such that $v_{1} \in J_{m_{2}} \backslash J_{m_{2}+1}$, and we can proceed inductively. At the end of this process we get sequences $m_{1}<m_{2}<\cdots<m_{N} \leq k+p$ (so $N \leq p$ ) and $v_{1}, v_{2}, \ldots, v_{N}$ with $v_{j} \in J_{m_{j}} \backslash J_{m_{j}+1}$, and for each $j$ an integer $k_{j}$ with $\left|k_{j}\right| \leq\left\lceil a_{m_{j}} / 2\right\rceil$ such that $v_{j+1}=f_{m_{j}}^{-k_{j}}\left(v_{j}\right)$. The last vertex $v_{N}$ is necessarily $x_{m}$ for some $m \leq k+p$. Hence it suffices to take $\phi_{j}=f_{m_{j}}^{k_{j}}$ to get the desired representation.

From now on, we assume that the corresponding sucessive renormalizations of $f$ and $g$ approach each other exponentially, in other words $\left\|\boldsymbol{f}_{n}-\boldsymbol{g}_{n}\right\|_{0} \leq C \mu^{n}$ for some $0<\mu<1$ and all $n \geq 0$, just as stated in the hypothesis of Lemma 4.7.

Lemma 4.10. There exists a constant $0<\mu_{*}<1$ for which the following holds. Let $v \in J_{k}(f)$ be a vertex of $\mathcal{P}_{k+p}(f)$ and let $w=h(v) \in J_{k}(g)$ be the corresponding vertex of $\mathcal{P}_{k+p}(g)$. If $\rho(f)$ satisfies condition (11), then we have

$$
|v-w| \leq C\left|J_{k}(f)\right| K^{p} \mu_{*}^{k},
$$

where $K \geq 1$ is the constant of Lemma 4.8.

Proof. By Lemma 4.9 above, there exist points $x_{m}=x_{m}(f), y_{m}=x_{m}(g)$ and a number $N \leq p$ such that

$$
|v-w|=\left|\phi_{1} \circ \phi_{2} \circ \cdots \circ \phi_{N}\left(x_{m}\right)-\psi_{1} \circ \psi_{2} \circ \cdots \circ \psi_{N}\left(y_{m}\right)\right|,
$$

where $\phi_{j}=f_{m_{j}}^{k_{j}}$ and $\psi_{j}=g_{m_{j}}^{k_{j}}$, with $k \leq m_{j} \leq k+p$ and $\left|k_{j}\right| \leq\left\lceil a_{m_{j}} / 2\right\rceil$. For each $i \geq 1$, let $\Lambda_{i, f}$ be the affine map $x \mapsto c+\left|I_{i}(f)\right| x$, and define $\Lambda_{i, g}$ in the same way. For each $i \geq k$, let $A_{i, f}=\Lambda_{k, f}^{-1} \circ \Lambda_{i, f}$ and $A_{i, g}=\Lambda_{k, g}^{-1} \circ \Lambda_{i, g}$. In order to estimate $|v-w|$, we shall estimate $\left|v^{*}-w^{*}\right|$, where $v^{*}=\Lambda_{k, f}^{-1}(v)$ and $w^{*}=\Lambda_{k, g}^{-1}(w)$. To do this, for each $i \geq k$ consider the map $f_{i}^{*}: \Lambda_{k, f}^{-1}\left(J_{i}(f)\right) \rightarrow \Lambda_{k, f}^{-1}\left(J_{i}(f)\right)$ given by

$$
f_{i}^{*}=\Lambda_{k, f}^{-1} \circ f_{i} \circ \Lambda_{k, f}=A_{i, f} \circ f_{i} \circ A_{i, f}^{-1},
$$

and let $g_{i}^{*}$ be similarly defined.

First we claim that for all $x \in \Lambda_{k, f}^{-1}\left(J_{i}(f)\right) \cap \Lambda_{k, g}^{-1}\left(J_{i}(g)\right)$ we have

$$
\left|f_{i}^{*}(x)-g_{i}^{*}(x)\right| \leq C_{1} \mu^{k} \frac{\left|I_{i}(f)\right|}{\left|I_{k}(f)\right|} .
$$


To see why, note that by inequality (13) of Lemma 4.7 we have, for all $z$ in the domain of both renormalizations $\boldsymbol{f}_{i}$ and $\boldsymbol{g}_{i}$,

$$
\begin{aligned}
\left|A_{i, f}(z)-A_{i, g}(z)\right| & =\left|\frac{\left|I_{i}(f)\right|}{\left|I_{k}(f)\right|}-\frac{\left|I_{i}(g)\right|}{\left|I_{k}(g)\right|}\right||z| \\
& \leq C_{2} \mu^{k} \frac{\left|I_{i}(f)\right|}{\left|I_{k}(f)\right|} .
\end{aligned}
$$

Similarly, for all $x \in \Lambda_{k, f}^{-1}\left(J_{i}(f)\right) \cap \Lambda_{k, g}^{-1}\left(J_{i}(g)\right)$ we have, again by (13),

$$
\begin{aligned}
\left|A_{i, f}^{-1}(x)-A_{i, g}^{-1}(x)\right| & =\left|\frac{\left|I_{k}(f)\right|}{\left|I_{i}(f)\right|}-\frac{\left|I_{k}(g)\right|}{\left|I_{i}(g)\right|}\right||x| \\
& \leq C_{3} \mu^{k} \frac{\left|I_{k}(f)\right|}{\left|I_{i}(f)\right|}|x| \leq C_{4} \mu^{k} .
\end{aligned}
$$

Here we have used that $|x| \leq\left|J_{i}(f)\right| /\left|I_{k}(f)\right| \leq C_{5}\left|I_{i}(f)\right| /\left|I_{k}(f)\right|$ (recall from the real bounds that $\left.\left|J_{i}(f)\right| \asymp\left|I_{i}(f)\right|\right)$. Also, by hypothesis we have $\left\|\boldsymbol{f}_{i}-\boldsymbol{g}_{i}\right\|_{0} \leq C_{6} \mu^{k}$. Combining these three estimates with a standard telescoping trick, we get (15), and the claim is proved.

Now let $\phi_{j}^{*}=A_{m_{j}, f} \circ \phi_{j} \circ A_{m_{j}, f}^{-1}$ and $\psi_{j}^{*}=A_{m_{j}, g} \circ \psi_{j} \circ A_{m_{j}, g}^{-1}$. Applying (15) with $i=m_{j}$ and using Proposition 4.1, we have

$$
\left|\phi_{j}^{*}(x)-\psi_{j}^{*}(x)\right| \leq C_{7}\left|k_{j}\right|^{3} \mu^{k} \frac{\left|I_{m_{j}}(f)\right|}{\left|I_{k}(f)\right|} .
$$

By the real bounds, there exists $0<\lambda_{1}<1$ such that $\left|I_{m_{j}}(f)\right| /\left|I_{k}(f)\right| \leq$ $C_{8} \lambda_{1}^{m_{j}-k}$. Taking $\lambda=\max \left\{\mu, \lambda_{1}\right\}$, we deduce from (16) that

$$
\left|\phi_{j}^{*}(x)-\psi_{j}^{*}(x)\right| \leq C_{9} a_{m_{j}}^{3} \lambda^{m_{j}} .
$$

We can at last start our estimate of $\left|v^{*}-w^{*}\right|$. First, note that $x_{m}=\Lambda_{m, f}(1)$ and $y_{m}=\Lambda_{m, g}(1)$. Writing $x_{m}^{*}=\Lambda_{k, f}^{-1}\left(x_{m}\right)$ and $y_{m}^{*}=\Lambda_{k, g}^{-1}\left(y_{m}\right)$, we see after a simple computation that $\left|x_{m}^{*}-y_{m}^{*}\right| \leq C_{10} \lambda^{m}$. Combining this fact with (17) and using Lemma 4.8, we get

$$
\begin{aligned}
\left|\phi_{N}^{*}\left(x_{m}^{*}\right)-\psi_{N}^{*}\left(y_{m}^{*}\right)\right| & \leq\left|\phi_{N}^{*}\left(x_{m}^{*}\right)-\psi_{N}^{*}\left(x_{m}^{*}\right)\right|+\left|\psi_{N}^{*}\left(x_{m}^{*}\right)-\psi_{N}^{*}\left(y_{m}^{*}\right)\right| \\
& \leq C_{9} a_{m_{N}}^{3} \lambda^{m_{N}}+C_{10} K \lambda^{m} .
\end{aligned}
$$

From this, and since

$$
\begin{aligned}
&\left|\phi_{N-1}^{*}\left(\phi_{N}^{*}\left(x_{m}^{*}\right)\right)-\psi_{N-1}^{*}\left(\psi_{N}^{*}\left(y_{m}^{*}\right)\right)\right| \leq \\
&\left|\phi_{N-1}^{*}\left(\phi_{N}^{*}\left(x_{m}\right)\right)-\psi_{N-1}^{*}\left(\phi_{N}^{*}\left(x_{m}^{*}\right)\right)\right|+\left|\psi_{N-1}^{*}\left(\phi_{N}^{*}\left(x_{m}^{*}\right)\right)-\psi_{N-1}^{*}\left(\psi_{N}^{*}\left(y_{m}^{*}\right)\right)\right|,
\end{aligned}
$$


we deduce that

$$
\begin{aligned}
\left|\phi_{N-1}^{*}\left(\phi_{N}^{*}\left(x_{m}^{*}\right)\right)-\psi_{N-1}^{*}\left(\psi_{N}^{*}\left(y_{m}^{*}\right)\right)\right| \leq \\
C_{9}\left(a_{m_{N-1}}^{3} \lambda^{m_{N-1}}+K a_{m_{N}}^{3} \lambda^{m_{N}}\right)+C_{10} K^{2} \lambda^{m} .
\end{aligned}
$$

Proceeding inductively in this fashion, we get in the end

$$
\left|v^{*}-w^{*}\right| \leq C_{9} \sum_{j=1}^{N} K^{j-1} a_{m_{j}}^{3} \lambda^{m_{j}}+C_{10} K^{N} \lambda^{m} .
$$

Using that $N \leq p$ and taking $C_{11}=\max \left\{C_{9}, C_{10}\right\}$, we arrive at

$$
\left|v^{*}-w^{*}\right| \leq C_{11} K^{p}\left(\lambda^{m}+\sum_{j=1}^{N} a_{m_{j}}^{3} \lambda^{m_{j}}\right) .
$$

We have of course $\lambda^{m} \leq \lambda^{k}$. Moreover, since $k \leq m_{j}<m_{j+1}$ for all $j$, we have

$$
\sum_{j=1}^{N} a_{m_{j}}^{3} \lambda^{m_{j}}<\sum_{n=k}^{\infty} a_{n}^{3} \lambda^{n} .
$$

But since $\left(a_{n}\right)$ satisfies condition (11), we know that $\lim \left(a_{n}^{3}\right)^{1 / n}=1$. In particular, if $\varepsilon>0$ is such that $(1+\varepsilon) \sqrt{\lambda}=1$, there exists $C_{12}=C_{12}(\varepsilon)>0$ such that $a_{n}^{3}<C_{12}(1+\varepsilon)^{n}$ for all $n$. Therefore

$$
\sum_{n=k}^{\infty} a_{n}^{3} \lambda^{n}<C_{12} \sum_{n=k}^{\infty}(\sqrt{\lambda})^{n}=\frac{C_{12}}{1-\sqrt{\lambda}}(\sqrt{\lambda})^{k} .
$$

Taking this back to (18) yields $\left|v^{*}-w^{*}\right| \leq C_{13} K^{p}(\sqrt{\lambda})^{k}$. Therefore, noting that under the assumption given in the remark after Lemma 4.7 we have

$$
|v-w|=\left|\Lambda_{k, f}\left(v^{*}\right)-\Lambda_{k, g}\left(w^{*}\right)\right| \leq\left|I_{k}(f)\right|\left(\left|v^{*}-w^{*}\right|+C_{14} \mu^{k}\right),
$$

and taking $\mu_{*}=\sqrt{\lambda}$, we get (14) as desired.

Lemma 4.11. There exists a constant $M>0$ depending only on the real bounds such that if $\Delta^{*} \in \mathcal{P}_{k}(f)$ and $\Delta \in \mathcal{P}_{k+p}(f)$ is contained in $\Delta^{*}$, then

$$
|\Delta| \geq \frac{M^{p}}{\left(a_{k+1} a_{k+2} \cdots a_{k+p}\right)^{2}}\left|\Delta^{*}\right| .
$$

Proof. This again follows from Yoccoz's Lemma and a simple inductive argument. 
Definition. The level of an atom $\Delta \in \mathcal{Q}_{n}(f)$, denoted $\ell(\Delta)$, is the largest $m \leq n$ such that $\Delta$ is contained in an atom of $\mathcal{P}_{m}(f)$.

Lemma 4.12. If $\mathcal{Q}_{n}(f)$ contains an atom of level $m$, then

$$
n \leq c_{0} \sum_{j=1}^{m} \log \left(1+a_{j}\right)
$$

for some absolute constant $c_{0}>0$. In particular, if the partial quotients of $\rho(f)$ satisfy (10), then $m \geq c_{1} n$ for some constant $0<c_{1}<1$ that depends only on $\rho(f)$.

Proof. Let $\Delta \in \mathcal{Q}_{n}(f)$ be an atom of level $m$. Let $\Delta_{1} \supseteq \Delta_{2} \supseteq \cdots \supseteq \Delta_{n}=\Delta$ be such that $\Delta_{k} \in \mathcal{Q}_{k}(f)$, and note that $1=\ell\left(\Delta_{1}\right) \leq \ell\left(\Delta_{2}\right) \leq \cdots \leq$ $\ell\left(\Delta_{n}\right)=m$. Given $1 \leq l \leq m$, let $i$ and $s$ (maximal) be such that

$$
\ell\left(\Delta_{i+1}\right)=\ell\left(\Delta_{i+2}\right)=\cdots=\ell\left(\Delta_{i+s}\right)=l .
$$

Then there exists $I \in \mathcal{P}_{l}(f)$ such that each $\Delta_{j}$ with $i+1 \leq j \leq i+s$ is a union of atoms of $\mathcal{P}_{l+1}(f)$ inside $I$. From the very construction of the partitions $\mathcal{Q}_{j}(f)$ (Proposition 4.5), we see that the number of atoms of $\mathcal{P}_{l+1}(f)$ inside $\Delta_{j}$ is at least twice the number of such atoms inside $\Delta_{j+1}$, for each $i+1 \leq j \leq i+s-1$. Moreover, $\Delta_{i+s}$ contains at least two such atoms (otherwise its level would be $l+1$ ). Since the total number of atoms of $\mathcal{P}_{l+1}(f)$ that lie inside $I$ is at most $1+a_{l}$, it follows that $2^{s} \leq 1+a_{l}$, whence $s \leq \log _{2}\left(1+a_{l}\right)$. This proves (19) with $c_{0}=1 / \log 2$.

Now, if $\rho(f)$ satisfies (10), then there exists $B>0$ depending on $\rho(f)$ such that $\sum_{j=1}^{m} \log a_{j} \leq B m$. Therefore

$$
n \leq c_{0} \sum_{j=1}^{m} \log \left(1+a_{j}\right) \leq c_{0}(B+\log 2) m,
$$

which proves the last assertion, with $c_{1}=c_{0}^{-1}(B+\log 2)^{-1}$.

Lemma 4.13. If $\rho(f)$ satisfies (11) and (12) then there exists $0<\beta<1$ with the following property. If $L$ and $R$ are adjacent atoms of $\mathcal{Q}_{n}(f)$ and we have $\ell(L) \geq m$ and $\ell(R) \geq m$, then

$$
\left|\frac{|L|}{|R|}-\frac{|h(L)|}{|h(R)|}\right| \leq C \beta^{m} .
$$

Proof. Write $m=k+p$ with $p=\lceil\sigma k\rceil$ where $\sigma>0$ is a small constant (its size will be determined in the course of the argument). We may assume that $L \cup R$ is contained in a single atom $\Delta$ of $\mathcal{P}_{k}(f)$. There are two cases to consider. 
(a) If $L \cup R \subseteq J_{k}(f)$, then the required coherence estimate (20) follows from Lemma 4.10 and Lemma 4.11. To see this, let $v_{1}, v_{2}, v_{3} \in \mathcal{P}_{k+p}(f)$ be the endpoints of $L$ and $R, v_{2}$ being their common endpoint. Let $w_{1}, w_{2}, w_{3}$ be the corresponding endpoints of $h(L)$ and $h(R)$. Then by Lemma 4.10 we have $\left|v_{i}-w_{i}\right| \leq C_{0}\left|J_{k}(f)\right| \theta^{k}$, where $\theta=K^{\sigma} \mu_{*}<1$ if $\sigma$ is small enough. On the other hand, condition (12) tells us that

$$
a_{k+1} a_{k+2} \cdots a_{k+p} \leq \exp \{p \omega(p / k)\} \leq \exp \{p \omega(\sigma)\} .
$$

Combining this fact with Lemma 4.11, we get

$$
\left|v_{1}-v_{2}\right| \geq \frac{M^{p}}{\left(a_{k+1} a_{k+2} \cdots a_{k+p}\right)^{2}}\left|J_{k}(f)\right| \geq \frac{M^{p}}{e^{2 p \omega(\sigma)}}\left|J_{k}(f)\right| .
$$

The same lower bound holds for $\left|v_{2}-v_{3}\right|$. From these facts, we deduce after some simple computations that

$$
\begin{aligned}
\left|\frac{|L|}{|R|}-\frac{|h(L)|}{|h(R)|}\right| & =\left|\frac{\left|v_{1}-v_{2}\right|}{\left|v_{2}-v_{3}\right|}-\frac{\left|w_{1}-w_{2}\right|}{\left|w_{2}-w_{3}\right|}\right| \\
& \leq C_{1} \frac{\theta^{k} e^{2 p \omega(\sigma)}}{M^{p}} \leq C_{2}\left(\frac{\theta e^{2 \sigma \omega(\sigma)}}{M^{\sigma}}\right)^{k} \leq C_{3} \beta_{1}^{m},
\end{aligned}
$$

where $\beta_{1}=\left(\theta e^{2 \sigma \omega(\sigma)} / M^{\sigma}\right)^{1 /(1+\sigma)}$. Since $\theta<1$ and $\sigma \omega(\sigma) \rightarrow 0$ as $\sigma \rightarrow 0$, we see that $\beta_{1}<1$ if $\sigma$ is small enough.

(b) If $L \cup R$ is not contained in $J_{k}(f)$, there exists $j<q_{k+1}$ such that $f^{j}$ is a diffeomorphism on an interval containing $\Delta$ and its two neighbors in $\mathcal{P}_{k}(f)$ and such that $f^{j}(\Delta) \subseteq J_{k}(f)$. By the Koebe principle and the real bounds, the distortion of $f^{j}$ on $L \cup R$ is bounded by $\exp \left(C_{4} \mu_{0}^{p}\right)$ (where $0<\mu_{0}<1$ is the beau constant of Theorem 3.1). Therefore we have

$$
\left|\frac{|L|}{|R|}-\frac{\left|f^{j}(L)\right|}{\left|f^{j}(R)\right|}\right| \leq C_{5} \mu_{0}^{p} \leq C_{6} \mu_{1}^{m},
$$

where $\mu_{1}=\mu_{0}^{\sigma /(1+\sigma)}$. Working similarly with $h(L), h(R) \in \mathcal{Q}_{n}(g)$, we get also

$$
\left|\frac{|h(L)|}{|h(R)|}-\frac{\left|g^{j}(h(L))\right|}{\left|g^{j}(h(R))\right|}\right| \leq C_{7} \mu_{1}^{m} .
$$

Putting (21) and (22) together and using (a) we get inequality (20) with the constant $\beta=\max \left\{\mu_{1}, \beta_{1}\right\}$.

Hence in both cases (20) is established, and we are done.

The proof of our First Main Theorem is now almost complete. If $L$ and $R$ are adjacent atoms of $\mathcal{Q}_{n}(f)$ as above, then combining Lemma 4.12 with Lemma 4.13 we deduce that the coherence condition (6) is satisfied with $\lambda=\beta^{c_{1}}$. Therefore by Proposition 4.3 the conjugacy $h$ is indeed $C^{1+\alpha}$ for some $\alpha>0$. 


\section{Counterexamples to $C^{1+\alpha}$ rigidity}

Our purpose now is to construct $C^{\infty}$ counterexamples to the conjectured $C^{1+\alpha}$ rigidity of critical circle maps. We will consider critical circle maps whose rotation number $\rho(f)=\left[a_{0}, a_{1}, \ldots, a_{n}, \ldots\right]$ satisfies

$$
\left\{\begin{array}{l}
\limsup \frac{1}{n} \log a_{n}=\infty \\
a_{n} \geq 2
\end{array} \text { for all } n .\right.
$$

The class of all rotation numbers satisfying (23) will be denoted by $\mathbb{B}$. It can be shown that the Hausdorff dimension of $\mathbb{B}$ is less than or equal to $1 / 2$, see [7]. On the other hand, $\mathbb{B}$ contains Diophantine numbers: for example, the number $\rho$ whose partial quotients are $a_{n}=2^{2^{n}}$ is Diophantine and satisfies (23).

Theorem 5.1. For every $\rho \in \mathbb{B}$ there exist $C^{\infty}$ critical circle maps $f, g$ with $\rho(f)=\rho(g)=\rho$ such that $f$ and $g$ are not $C^{1+\beta}$ conjugate for any $\beta>0$.

The proof will make use of a $C^{\infty}$ surgery procedure that we explain below. These counterexamples have one additional feature: their successive renormalizations do converge together at an exponential rate. This will be clear from the construction.

\subsection{Saddle-node surgery}

Given $f$ as above and a fixed $n \geq 1$, let $J_{n}=J_{n}(f)=\left[f^{q_{n}}(c), f^{q_{n-1}}(c)\right] \subseteq$ $S^{1}$ be the $n$-th renormalization interval of $f$. When $a_{n}$ is very large, the first return map $f_{n}: J_{n} \rightarrow J_{n}$ is an almost parabolic map of length $a_{n}$.

Let $\Delta_{1}^{(n)}$ be the fundamental domain of this almost parabolic map which is adjacent to $x_{n-1}=f^{q_{n-1}}(c)$, and let $\Delta_{j}^{(n)}=f_{n}^{j-1}\left(\Delta_{1}^{(n)}\right), j \leq a_{n}$. Let $z_{n} \in \Delta_{1}^{(n)}$ be the point such that $f_{n}^{a_{n}}\left(z_{n}\right)=x_{n+2}=f^{q_{n+2}}(c)$, that is, $z_{n}=f^{q_{n+2}-a_{n} q_{n}}(c)$. Note that since $a_{n} \geq 2, x_{n+2}$ is not an endpoint of $f_{n}^{a_{n}}\left(\Delta_{1}^{(n)}\right)$, and so by the real bounds it splits $f_{n}^{a_{n}}\left(\Delta_{1}^{(n)}\right)$ into two intervals of comparable lengths. Hence the same holds for $z_{n}$. Namely, $z_{n}$ splits $\Delta_{1}^{(n)}$ into two intervals $L_{n}, R_{n}$ with $\left|L_{n}\right| \asymp\left|R_{n}\right|$. In particular we have $\tau\left|\Delta_{1}^{(n)}\right| \leq\left|L_{n}\right| \leq(1-\tau)\left|\Delta_{1}^{(n)}\right|$ (and similarly for $R_{n}$ ) for some constant $\tau$ depending on the real bounds. We use this fact in the proof of Proposition 5.2 below.

Consider now another critical circle map $\tilde{f}$ with the same rotation number as $f$, the interval $\tilde{J}_{n}=J_{n}(\tilde{f})$, the first return map $\tilde{f}_{n}: \tilde{J}_{n} \rightarrow \tilde{J}_{n}$, the point $\tilde{z}_{n}=\tilde{f}^{q_{n+2}-a_{n} q_{n}}(\tilde{c})$ and the corresponding intervals $\tilde{L}_{n}, \tilde{R}_{n}$. Also, let $N_{n}=\left\lceil a_{n} / 2\right\rceil$. 
Definition. The number

$$
\left|\frac{\left|f_{n}^{N_{n}-1}\left(L_{n}\right)\right|}{\left|f_{n}^{N_{n}-1}\left(R_{n}\right)\right|}-\frac{\left|\tilde{f}_{n}^{N_{n}-1}\left(\tilde{L}_{n}\right)\right|}{\left|\tilde{f}_{n}^{N_{n}-1}\left(\tilde{R}_{n}\right)\right|}\right|
$$

is called the $n$-th order discrepancy between $f$ and $\tilde{f}$.

Proposition 5.2. Given a $C^{\infty}$ critical circle map $f$ with $\rho(f) \in \mathbb{B}$, consider a function $\sigma(n) \rightarrow \infty$ such that

$$
\lim \sup \frac{1}{n \sigma(n)} \log a_{n}=\infty \text {. }
$$

Then for all $n \geq 1$, there exists a critical circle map $\tilde{f}=F(n ; f)$ with the same rotation number and critical point as $f$ and having the following properties.

(a) We have $\tilde{f}^{j}(c)=f^{j}(c)$ for $0 \leq j \leq q_{n+1}$; in particular, $J_{n}(\tilde{f})=J_{n}=$ $J_{n}(f)$.

(b) We have $\tilde{f}=\Phi \circ f$, where $\Phi$ is a $C^{\infty}$ diffeo such that

$$
\left\|\Phi^{ \pm 1}-i d_{S^{1}}\right\|_{C^{k}} \leq B_{k}\left|J_{n}\right|^{\sigma(n)-k+1}
$$

for all $k$, where $B_{k}>0$ is constant depending only on $k$.

(c) The $n$-th order discrepancy between $f$ and $\tilde{f}$ is $\geq C\left|J_{n}\right|^{2 \sigma(n)}$.

(d) We have $J_{n+1}(\tilde{f})=J_{n+1}(f)$ and $\tilde{f}_{n+1}=f_{n+1}$; in particular, $m$-th order discrepancy between $f$ and $\tilde{f}$ is equal to zero for all $m>n$.

Proof. We modify $f$ inside $f^{-1}\left(\Delta_{1}^{(n)}\right)$ using a $C^{\infty}$ bump function so as to move $z_{n}$ by a distance $\geq C\left|\Delta_{1}^{(n)}\right|^{1+\sigma(n)}$ inside $\Delta_{1}^{(n)}$. This we do as follows.

Let $\varphi:[0,1] \rightarrow[0,1]$ be a $C^{\infty}$ perturbation of the identity such that $|\varphi(x)-x| \geq\left|\Delta_{1}^{(n)}\right|^{\sigma(n)}$ for all $\tau \leq x \leq 1-\tau$ (and $\tau$ as above), and such that $\left|D^{k} \varphi(x)\right| \leq B_{k}\left|\Delta_{1}^{(n)}\right|^{\sigma(n)}$ for all $0 \leq x \leq 1$ and all $k \geq 2$. Define $\phi_{n}: \Delta_{1}^{(n)} \rightarrow \Delta_{1}^{(n)}$ by $\phi_{n}=A_{n} \circ \varphi \circ A_{n}^{-1}$ where $A_{n}$ is the affine orientationpreserving map that carries $[0,1]$ onto $\Delta_{1}^{(n)}$. Note that $\left|\phi_{n}\left(z_{n}\right)-z_{n}\right| \geq$ $\left|\Delta_{1}^{(n)}\right|^{1+\sigma(n)}$. Moreover, since $D^{k} \phi_{n}=\left|\Delta_{1}^{(n)}\right|^{1-k} D^{k} \varphi$, we have

$$
\left\|\phi_{n}^{ \pm 1}-\mathrm{id}_{\Delta_{1}^{(n)}}\right\|_{C^{k}} \leq B_{k}\left|\Delta_{1}^{(n)}\right|^{\sigma(n)-k+1}
$$

for all $k$. Define $\psi_{n}: \Delta_{a_{n}}^{(n)} \rightarrow \Delta_{a_{n}}^{(n)}$ as the conjugate of $\phi_{n}^{-1}$ by the diffeo $f_{n}^{a_{n}-1}: \Delta_{1}^{(n)} \rightarrow \Delta_{a_{n}}^{(n)}$, namely

$$
\psi_{n}=f_{n}^{a_{n}-1} \circ \phi_{n}^{-1} \circ\left(f_{n}^{a_{n}-1}\right)^{-1} .
$$


Using the $C^{m}$ Approximation Lemma (see Appendix A), we see from (24) that

$$
\left\|\psi_{n}^{ \pm 1}-\mathrm{id}_{\Delta_{a_{n}}^{(n)}}\right\|_{C^{k-1}} \leq C\left\|\phi_{n}^{ \pm 1}-\mathrm{id}_{\Delta_{1}^{(n)}}\right\|_{C^{k}} \leq B_{k}\left|\Delta_{1}^{(n)}\right|^{\sigma(n)-k+1} .
$$

Define $\Phi: S^{1} \rightarrow S^{1}$ to be equal to $\phi_{n}$ on $\Delta_{1}^{(n)}$, to $\psi_{n}$ on $\Delta_{a_{n}}^{(n)}$ and to the identity everywhere else. The critical circle map we look for is $\tilde{f}=\Phi \circ f$. Note that $\left\|\Phi^{ \pm 1}-\mathrm{id}_{S^{1}}\right\|_{C^{k}} \leq B_{k}\left|\Delta_{1}^{(n)}\right|^{\sigma(n)-k+1}$ for all $k$; since $\left|\Delta_{1}^{(n)}\right| \asymp\left|J_{n}\right|$ by the real bounds, this proves $(b)$. It is also clear from the construction that property $(a)$ holds too. It follows in particular that the first $n+1$ partial quotients of the rotation number of $\tilde{f}$ agree with those of $f$. More remarkable is that, because what $\phi_{n}$ does is undone by $\psi_{n}$, we have

$$
\left\{\begin{array}{l}
\tilde{f}^{q_{n}}\left|I_{n+1}=f^{q_{n}}\right| I_{n+1} \\
\tilde{f}^{q_{n+1}}\left|I_{n}=f^{q_{n+1}}\right| I_{n}
\end{array} .\right.
$$

In other terms, $\tilde{f}_{n}=f_{n}$, the $n$-th renormalizations agree. Therefore all subsequent renormalizations agree as well. This shows that $\rho(\tilde{f})=\rho(f)$ and also proves $(d)$.

It remains to prove (c), so we estimate the $n$-th order discrepancy between $f$ and $\tilde{f}$ from below. Since $\left|z_{n}-\tilde{z}_{n}\right| \geq\left|\Delta_{1}^{(n)}\right|^{1+\sigma(n)}$, a simple calculation yields

$$
\left|\frac{\left|L_{n}\right|}{\left|R_{n}\right|}-\frac{\left|\tilde{L}_{n}\right|}{\left|\tilde{R}_{n}\right|}\right| \geq C\left|\Delta_{1}^{(n)}\right|^{\sigma(n)} \geq C\left|J_{n}\right|^{2 \sigma(n)},
$$

provided $n$ is sufficiently large. Since, by the real bounds, the map $f_{n}^{N-1}: \Delta_{1}^{(n)} \rightarrow \Delta_{N_{n}}^{(n)}$ has bounded distortion, and since $\tilde{f}_{n}=f_{n}$, inequality (25) gives us

$$
\left|\frac{\left|f_{n}^{N_{n}-1}\left(L_{n}\right)\right|}{\left|f_{n}^{N_{n}-1}\left(R_{n}\right)\right|}-\frac{\left|\tilde{f}_{n}^{N_{n}-1}\left(\tilde{L}_{n}\right)\right|}{\left|\tilde{f}_{n}^{N_{n}-1}\left(\tilde{R}_{n}\right)\right|}\right| \geq C\left|J_{n}\right|^{2 \sigma(n)},
$$

and this proves $(c)$.

\subsection{The counterexamples}

We now iterate the procedure given by Proposition 5.2 to prove our Second Main Theorem (that is, Theorem 5.1). We start with a $C^{\infty}$ map $f$ with $\rho(f) \in \mathbb{B}$ as before and select $n_{1}<n_{2}<\cdots$ such that

$$
\lim _{i \rightarrow \infty} \frac{1}{n_{i} \sigma\left(n_{i}\right)} \log a_{n_{i}}=\infty
$$


where $\sigma(n)$ is as in Proposition 5.2. Now we generate a sequence $g_{0}$, $g_{1}, \ldots, g_{i}, \ldots$ recursively, starting with $g_{0}=f$, and taking, for all $i \geq 0$, $g_{i+1}=F\left(n_{i+1}, g_{i}\right)$, where $F(\cdot, \cdot)$ is as given in Proposition 5.2. Each $g_{i}$ is a $C^{\infty}$ critical circle map with $\rho\left(g_{i}\right)=\rho(f)$, and $g_{i+1}=\Phi_{i+1} \circ g_{i}$, where $\Phi_{i+1}$ is a $C^{\infty}$ diffeo with

$$
\left\|\Phi_{i+1}^{ \pm 1}-\mathrm{id}_{S^{1}}\right\|_{C^{k}} \leq B_{k} \theta^{n_{i}\left(\sigma\left(n_{i}\right)-k+1\right)},
$$

for all $k$, where $0<\theta<1$ is a constant depending only on the real bounds. From (27) it follows that $\Phi=\lim \Phi_{i} \circ \cdots \circ \Phi_{1}$ exists as a $C^{\infty}$ diffeo, and therefore so does $g=\lim g_{i}=\Phi \circ f$ as a critical circle map.

Using properties $(c)$ and $(d)$ of Proposition 5.2 for each $g_{i}$, we deduce that the $n_{i}$-th order discrepancy between $f$ and $g$ satisfies

$$
\left|\frac{\left|f_{n_{i}}^{N_{i}-1}\left(L_{n_{i}}\right)\right|}{\left|f_{n_{i}}^{N_{i}-1}\left(R_{n_{i}}\right)\right|}-\frac{\left|g_{n_{i}}^{N_{i}-1}\left(\tilde{L}_{n_{i}}\right)\right|}{\left|g_{n_{i}}^{N_{i}-1}\left(\tilde{R}_{n_{i}}\right)\right|}\right| \geq C\left|J_{n_{i}}\right|^{2 \sigma\left(n_{i}\right)},
$$

where $N_{i}=\left\lceil a_{n_{i}} / 2\right\rceil$, etc.

Now, let $h: S^{1} \rightarrow S^{1}$ be the conjugacy between $f$ and $g$ mapping the critical point $c$ to itself. Suppose $h$ were $C^{1+\beta}$ for some $\beta>0$. Then the lefthand side of (28) would be $\leq C\left|f_{n_{i}}^{N_{i}-1}\left(\Delta_{1}^{\left(n_{i}\right)}\right)\right|^{\beta}$, where $\Delta_{1}^{\left(n_{i}\right)}=L_{n_{i}} \cup R_{n_{i}}$. But by Yoccoz's Lemma, we have

$$
\left|f_{n_{i}}^{N_{i}-1}\left(\Delta_{1}^{\left(n_{i}\right)}\right)\right| \asymp \frac{1}{N_{i}^{2}}\left|J_{n_{i}}\right| \asymp \frac{1}{a_{n_{i}}^{2}}\left|J_{n_{i}}\right| .
$$

Combining the above with (28) and (29), we would get the inequality

$$
a_{n_{i}}^{2 \beta}\left|J_{n_{i}}\right|^{2 \sigma\left(n_{i}\right)-\beta} \leq C .
$$

But by the real bounds $\left|J_{n}\right| \geq C \mu^{n}$ for all $n$, where $0<\mu<1$. Therefore, taking logarithms, we would have

$$
\limsup \frac{\log a_{n_{i}}}{n_{i} \sigma\left(n_{i}\right)} \leq \frac{1}{\beta} \log \frac{1}{\mu},
$$

but this clearly contradicts (26).

Remark. A closer look at the construction performed above, especially at expressions (27) and (30), reveals that if

$$
\limsup \frac{1}{n} \log a_{n}>\frac{k}{\beta_{0}} \log \frac{1}{\mu}
$$

then one can construct a pair of $C^{k}$ critical circle maps (whose renormalizations converge exponentially fast) that are not $C^{1+\beta}$ conjugate for any $\beta \geq \beta_{0}$. 


\section{Appendix A. Compactness of renormalizations}

The real a-priori bounds proved in the Sect. 3 have produced a very important corollary, namely, that the renormalizations of an arbitrary $C^{3}$ critical circle map are uniformly bounded in the $C^{1}$ topology. In this appendix we will use further a-priori estimates, this time involving the Schwarzian derivative, to prove that such renormalizations are uniformly bounded in the $C^{r-1}$ topology when the critical circle map is $C^{r}$. Some technical tools are necessary.

\section{A.1. The $C^{m}$-Approximation Lemma}

In what follows, $m \geq 1$ will be a fixed integer and $I, J \subseteq \mathbb{R}$ fixed closed intervals. We denote by $C^{m}(I)$ the Banach space of $C^{m}$-mappings $f: I \rightarrow \mathbb{R}$ with the norm $\|f\|_{m}=\max \left\{\left\|D^{i} f\right\|_{0}: 0 \leq i \leq m\right\}$, where $\|\phi\|_{0}=\sup _{x \in I}|\phi(x)|$. Sometimes, when we need to emphasize the domain of $f$, we write $\|f\|_{I, m}$ instead of $\|f\|_{m}$. We consider also the closed, convex subset $C^{m}(I, J) \subseteq C^{m}(I)$ consisting of those $f$ 's such that $f(I) \subseteq J$.

Recall Leibnitz's formula for the $k$-th derivative of a product of two functions,

$$
D^{k}(u v)=\sum_{j=0}^{k}\left(\begin{array}{l}
k \\
j
\end{array}\right) D^{j} u D^{k-j} v
$$

from which it is clear that

$$
\|u v\|_{m} \leq 2^{m}\|u\|_{m}\|v\|_{m}
$$

whenever $u, v \in C^{m}(I)$. Something similar holds for the composition of two $C^{m}$ mappings. Namely, we have Faa-di-Bruno's formula (cf. [11], p. 42)

$$
D^{k}(f \circ g)=\sum_{j=1}^{k} B_{j, k}\left(D^{1} g, D^{2} g, \ldots, D^{j} g\right) D^{k-j+1} f \circ g,
$$

where each $B_{j, k}$ is a homogeneous polynomial of degree $k-j+1$ on $j$ variables whose coefficients are non-negative numbers depending only on $k$ and $j$. It readily follows from this formula that if $\psi \in C^{m}(I, J)$ and $\phi \in C^{m}(J)$ then

$$
\|\phi \circ \psi\|_{m} \leq A(m)\|\phi\|_{m} \sum_{k=1}^{m}\|\psi\|_{m}^{k},
$$

where $A(m)=\max _{1 \leq k \leq m} \max _{1 \leq j \leq k} B_{j, k}(1,1, \ldots, 1)$.

Another well-known fact we will need below is the following ( $c f$. [6], Th. 3.1). Suppose $m>1$ and consider the composition operator 
$(f, g) \mapsto f \circ g$ as a map $\Theta: C^{m}(J) \times C^{m-1}(I, J) \rightarrow C^{m-1}(I)$. Then $\Theta$ is $C^{1}$ and its Fréchet derivative is given by

$$
D \Theta(f, g)(u, v)=u \circ g+v D f \circ g .
$$

Note that $C^{m}(J) \times C^{m-1}(I, J) \subseteq C^{m}(J) \times C^{m-1}(I)$; we consider this last product endowed with the norm

$$
|(f, g)|_{I, J, m}=\max \left\{\|f\|_{J, m},\|g\|_{I, m-1}\right\} .
$$

Lemma A.1. For each $M>0$, there exists $c(M)>0$ such that, if $f_{1}, g_{1} \in$ $C^{m}(J)$ and $f_{2}, g_{2} \in C^{m-1}(I, J)$ and if $\left|\left(f_{1}, f_{2}\right)\right|_{I, J, m}<M$ and $\left|\left(g_{1}, g_{2}\right)\right|_{I, J, m}<$ $M$, then

$$
\left\|f_{1} \circ f_{2}-g_{1} \circ g_{2}\right\|_{m-1} \leq c(M)\left|\left(f_{1}-g_{1}, f_{2}-g_{2}\right)\right|_{I, J, m} .
$$

Proof. By the mean value theorem,

$$
\left\|f_{1} \circ f_{2}-g_{1} \circ g_{2}\right\|_{m-1} \leq \sup _{(\phi, \psi)}\|D \Theta(\phi, \psi)\|\left|\left(f_{1}-g_{1}, f_{2}-g_{2}\right)\right|_{I, J, m},
$$

where the supremum is taken over all $(\phi, \psi)$ in the line segment joining $\left(f_{1}, f_{2}\right)$ to $\left(g_{1}, g_{2}\right)$ inside $C^{m}(J) \times C^{m-1}(I, J)$, and where

$$
\|D \Theta(\phi, \psi)\|=\sup \left\{\|D \Theta(\phi, \psi)(u, v)\|_{m-1}:|(u, v)|_{I, J, m} \leq 1\right\}
$$

is the operator-norm of $D \Theta(\phi, \psi)$. Using (33), and then (31) and (32), we have

$$
\begin{aligned}
& \|D \Theta(\phi, \psi)(u, v)\|_{m-1} \leq\|u \circ \psi\|_{m-1}+\|v D \phi \circ \psi\|_{m-1} \\
& \leq\left(\|u\|_{m-1}+2^{m-1}\|v\|_{m-1}\|D \phi\|_{m-1}\right) A(m-1) \sum_{k=1}^{m-1}\|\psi\|_{m-1}^{k} .
\end{aligned}
$$

From this, and taking into account that $\|u\|_{m-1} \leq\|u\|_{m} \leq|(u, v)|_{I, J, m}$ as well as $\|v\|_{m-1} \leq|(u, v)|_{I, J, m}$, we deduce that

$$
\|D \Theta(\phi, \psi)\| \leq A(m-1)\left(1+2^{m-1}\|D \phi\|_{m-1}\right) \sum_{k=1}^{m-1}\|\psi\|_{m-1}^{k} .
$$

Finally, since $\|D \phi\|_{m-1} \leq\|\phi\|_{m}$ and $|(\phi, \psi)|_{I, J, m}<M$, we get

$$
\sup _{(\phi, \psi)}\|D \Theta(\phi, \psi)\| \leq A(m-1)\left(1+2^{m-1} M\right) \sum_{k=1}^{m-1} M^{k}=c(M) .
$$

Let us denote by $\mathbb{B}^{m}(I ; M)$ the ball of radius $M$ centered at the origin in $C^{m}(I)$. 
Lemma A.2. (The $C^{m}$-Approximation Lemma)

For each $M>0$, there exist constants $\varepsilon_{M}>0$ and $C_{M}>0$ such that the following holds for all $\varepsilon \leq \varepsilon_{M}$. Let $\Delta_{1}, \Delta_{2}, \ldots, \Delta_{n+1}$ be closed intervals on the line or on the circle, and for each $1 \leq i \leq n$ let $f_{i}, g_{i} \in C^{m}\left(\Delta_{i}, \Delta_{i+1}\right)$ be such that

(a) For all $1 \leq j \leq k \leq n$, we have $f_{k} \circ f_{k-1} \circ \cdots \circ f_{j} \in \mathbb{B}^{m}\left(\Delta_{j} ; M\right)$;

(b) We have $\sum_{i=1}^{n}\left\|f_{i}-g_{i}\right\|_{m}<\varepsilon$.

Then for all $k \leq n$ we have $g_{k} \circ g_{k-1} \circ \cdots \circ g_{1} \in \mathbb{B}^{m-1}\left(\Delta_{1} ; 2 M\right)$, and moreover

$\left\|f_{k} \circ f_{k-1} \circ \cdots \circ f_{1}-g_{k} \circ g_{k-1} \circ \cdots \circ g_{1}\right\|_{m-1} \leq C_{M} \sum_{j=1}^{k}\left\|f_{j}-g_{j}\right\|_{m}$.

Proof. In the notation of Lemma A.1, let us write

$$
C_{M}=\max \{1, c(2 M), c(2 M) c(3 M)\}
$$

and $\varepsilon_{M}=M / C_{M}$. We proceed by induction on $k$. When $k=1$, we have $\left\|f_{1}-g_{1}\right\|_{m} \leq \varepsilon$ and there is nothing to prove. Suppose the assertion is valid for all $j<k$, and write (omitting the composition symbols)

$$
\begin{aligned}
& \left\|f_{k} f_{k-1} \cdots f_{1}-g_{k} g_{k-1} \cdots g_{1}\right\|_{m-1} \leq \\
& \quad \sum_{j=1}^{k}\left\|f_{k} \cdots f_{j+1} g_{j} g_{j-1} \cdots g_{1}-f_{k} \cdots f_{j+1} f_{j} g_{j-1} \cdots g_{1}\right\|_{m-1} .
\end{aligned}
$$

Since $\left|\left(f_{j}, g_{j-1} \circ \cdots \circ g_{1}\right)\right|_{\Delta_{1}, \Delta_{j}, m}<2 M$ and also $\mid\left(g_{j}, g_{j-1} \circ\right.$ $\left.\cdots \circ g_{1}\right)\left.\right|_{\Delta_{1}, \Delta_{j}, m}<2 M$, it follows from Lemma A.1 that

$$
\left\|f_{j} g_{j-1} \cdots g_{1}-g_{j} g_{j-1} \cdots g_{1}\right\|_{m-1} \leq c(2 M)\left\|f_{j}-g_{j}\right\|_{m},
$$

for $j=1, \ldots, k$. In particular, by the induction hypothesis, we have for all $1 \leq j \leq k-1$

$$
\left\|f_{j} g_{j-1} \cdots g_{1}\right\|_{m-1} \leq\left\|g_{j} g_{j-1} \cdots g_{1}\right\|_{m-1}+\varepsilon_{M} c(2 M)<3 M .
$$

Taking this back to (34) and applying Lemma A.1 again, we get

$$
\begin{aligned}
\| f_{k} f_{k-1} \cdots f_{1} & -g_{k} g_{k-1} \cdots g_{1} \|_{m-1} \\
& \leq c(2 M)\left\|f_{k}-g_{k}\right\|_{m}+c(2 M) c(3 M) \sum_{j=1}^{k-1}\left\|f_{j}-g_{j}\right\|_{m} \\
& \leq C_{M} \sum_{j=1}^{k}\left\|f_{j}-g_{j}\right\|_{m},
\end{aligned}
$$

and this shows also that $\left\|g_{k} g_{k-1} \cdots g_{1}\right\|_{m-1} \leq M+\varepsilon_{M} C_{M}<2 M$, thereby completing the induction. 


\section{A.2. Koebe principle revisited}

We present a generalization of the classical Koebe non-linearity principle. This principle states that if a $C^{3}$ diffeomorphism has non-negative Schwarzian derivative on an open interval, then its non-linearity on any smaller closed subinterval with space on both sides is bounded. The generalized version below seems to be new. We denote by $S \phi$ the Schwarzian derivative of $\phi$.

Lemma A.3. Given positive constants $B$ and $\tau$, there exists $K_{\tau, B}>0$ such that the following holds. If $\phi$ is a $C^{3}$-diffeomorphism of an interval $I \supseteq[-\tau, 1+\tau]$ into the reals and if $S \phi(t) \geq-B$ for all $t \in I$, then for all $t \in[0,1]$ we have

$$
\left|\frac{\phi^{\prime \prime}(t)}{\phi^{\prime}(t)}\right| \leq K_{\tau, B} .
$$

Proof. Writing $y=\phi^{\prime \prime} / \phi^{\prime}$, so that $S \phi=y^{\prime}-\frac{1}{2} y^{2}$, we have the differential inequality

$$
y^{\prime} \geq \frac{1}{2} y^{2}-B .
$$

Let $0 \leq t_{0} \leq 1$ be a point where $|y(t)|$ attains its maximum in $[0,1]$ and suppose $y_{0}=y\left(t_{0}\right)$ is such that $\left|y_{0}\right|>\sqrt{2 B}=\beta$. If $z(t)$ is the solution of the differential equation corresponding to (35) with initial condition $z\left(t_{0}\right)=y_{0}$, then by a well-known comparison theorem we must have $y(t) \geq z(t)$ for all $t \geq t_{0}$ and $y(t) \leq z(t)$ for all $t \leq t_{0}$. Now, if $y_{0}>\beta$ then integration of the ODE leads to

$$
z(t)=\beta \frac{\left(y_{0}+\beta\right)+\left(y_{0}-\beta\right) e^{\beta\left(t-t_{0}\right)}}{\left(y_{0}+\beta\right)-\left(y_{0}-\beta\right) e^{\beta\left(t-t_{0}\right)}} .
$$

Since this solution explodes at time

$$
t_{1}=t_{0}+\frac{1}{\beta} \log \left(\frac{y_{0}+\beta}{y_{0}-\beta}\right),
$$

so does $y(t)$. Hence $t_{1} \notin I$, i.e. $t_{1}-t_{0}>\tau$, which gives us

$$
\frac{\phi^{\prime \prime}\left(t_{0}\right)}{\phi^{\prime}\left(t_{0}\right)}=y_{0}<\beta \frac{e^{\beta \tau}+1}{e^{\beta \tau}-1} .
$$

If instead $y_{0}<-\beta$, then we get

$$
z(t)=\beta \frac{\left(\beta+y_{0}\right)-\left(\beta-y_{0}\right) e^{\beta\left(t-t_{0}\right)}}{\left(\beta+y_{0}\right)+\left(\beta-y_{0}\right) e^{\beta\left(t-t_{0}\right)}},
$$

and arguing as before for $t \leq t_{0}$ gives us

$$
\frac{\phi^{\prime \prime}\left(t_{0}\right)}{\phi^{\prime}\left(t_{0}\right)}=y_{0}>-\beta \frac{e^{\beta \tau}+1}{e^{\beta \tau}-1} .
$$


Therefore the lemma is proved if we take

$$
K_{\tau, B}=\beta \frac{e^{\beta \tau}+1}{e^{\beta \tau}-1} .
$$

Remark. As $B \rightarrow 0, K_{\tau, B} \rightarrow 2 / \tau$ and we recover the classical Koebe principle.

\section{A.3. Bounding the $C^{2}$ norms}

As before, let $f \in \operatorname{Crit}^{r}\left(S^{1}\right), r \geq 3$, be a critical circle map with critical point $c$ of power-law $p>1$. Conjugating $f$ by a suitable $C^{r}$-diffeomorphism, we may assume that there exists a neighborhood $\mathcal{U} \subseteq \mathbb{R} / \mathbb{Z}$ of $c$ such that

$$
f(x)=(x-c)|x-c|^{p-1}+a
$$

for all $x \in \mathcal{U}$, where $a$ is a constant. This will be our standing hypothesis on $f$, and we will sometimes say that $f$ is a canonical circle map. Note in this case that for all $x \in \mathcal{U} \backslash\{c\}$, the Schwarzian derivative of $f$ equals

$$
S f(x)=-\frac{p^{2}-1}{2(x-c)^{2}} .
$$

We are interested in the maps $f^{q_{n-1}-1}: I_{n}^{1} \rightarrow I_{n}^{q_{n-1}}$ and $f^{q_{n}-1}: I_{n-1}^{1} \rightarrow$ $I_{n-1}^{q_{n}}$, for a fixed $n \geq 1$. They extend as diffeomorphisms to maximal open intervals $J_{n, 1}^{-} \supseteq I_{n}^{1}$ and $J_{n, 1}^{+} \supseteq I_{n-1}^{1}$ respectively. When linearly rescaled to unit size, these diffeomorphisms are called the coefficients of the $n$-th renormalization of $f$.

Let us be more precise. Consider the $n$-th renormalization of $f$, namely the commuting pair $f_{n}:\left[\lambda_{n}, 1\right] \rightarrow \mathbb{R}$ defined in Sect. 3. We write $J_{n, i}^{-}=$ $f^{i-1}\left(J_{n, 1}^{-}\right)$for each $1 \leq i \leq q=q_{n-1}$ and $J_{n, j}^{+}=f^{j-1}\left(J_{n, 1}^{+}\right)$for each $1 \leq j \leq Q=q_{n}$. We also write $J_{n, 0}^{-}=f^{-1}\left(J_{n, 1}^{-}\right)$and $J_{n, 0}^{+}=f^{-1}\left(J_{n, 1}^{+}\right)$. For each $0 \leq j \leq Q$, let $\Lambda_{j}: \mathbb{R} \rightarrow \mathbb{R} / \mathbb{Z}$ be the affine (orientation-preserving) covering map such that $\Lambda_{j}([0,1])=I_{n-1}^{j}$. Let $\Delta_{n}^{-}$be the component of $\Lambda_{1}^{-1}\left(J_{n, 1}^{-}\right)$that contains the interval $\left[\lambda_{n}, 0\right]$, and let $\Delta_{n}^{+}$be the component of $\Lambda_{1}^{-1}\left(J_{n, 1}^{+}\right)$that contains the interval $[0,1]$. Then define

$$
\left\{\begin{array}{l}
\mathcal{F}_{n}^{-}=\Lambda_{0}^{-1} \circ f^{q-1} \circ \Lambda_{1}: \Delta_{n}^{-} \rightarrow \mathbb{R} \\
\mathcal{F}_{n}^{+}=\Lambda_{0}^{-1} \circ f^{Q-1} \circ \Lambda_{1}: \Delta_{n}^{+} \rightarrow \mathbb{R}
\end{array} .\right.
$$

These are the $n$-th renormalization coefficients of $f$. Consider also the so-called folding factors of $\boldsymbol{f}_{n}$, namely the maps

$$
\left\{\begin{array}{l}
\varphi_{n}^{-}=\Lambda_{1}^{-1} \circ f \circ \Lambda_{0}: \Lambda_{0}^{-1}\left(J_{n, 0}^{-}\right) \rightarrow \mathbb{R} \\
\varphi_{n}^{+}=\Lambda_{1}^{-1} \circ f \circ \Lambda_{0}: \Lambda_{0}^{-1}\left(J_{n, 0}^{+}\right) \rightarrow \mathbb{R}
\end{array} .\right.
$$


Each of these maps is a homeomorphism with a unique critical point at zero. One verifies at once that the maps $\boldsymbol{F}_{n}^{-}=\mathcal{F}_{n}^{-} \circ \varphi_{n}^{-}$and $\boldsymbol{F}_{n}^{+}=\mathcal{F}_{n}^{+} \circ \varphi_{n}^{+}$are $C^{r}$ extensions of $\boldsymbol{f}_{n}^{-}$and $\boldsymbol{f}_{n}^{+}$, respectively.

It will be useful to express the coefficients $\mathcal{F}_{n}^{ \pm}$as long compositions of rescaled diffeomorphisms in the following way. We will give the explicit decomposition for $\mathcal{F}_{n}^{+}$. A similar decomposition can be worked out for $\mathcal{F}_{n}^{-}$. Let us denote by $\Delta_{n, j}^{+}$the component of $\Lambda_{j}^{-1}\left(J_{n, j}^{+}\right)$containing the unit interval. Note in particular that $\Delta_{n}^{+}=\Delta_{n, 1}^{+}$. For each $j$ in the range $0 \leq$ $j \leq Q-1$, let

$$
f_{j}=\Lambda_{j+1}^{-1} \circ f \circ \Lambda_{j}: \Delta_{n, j}^{+} \rightarrow \Delta_{n, j+1}^{+} .
$$

We call such maps the elementary factors of $\mathcal{F}_{n}^{+}$. Each $f_{j}$ is a $C^{r}$ diffeomorphism such that $f_{j}([0,1])=[0,1]$ (see Fig. 5). We have of course $\varphi_{n}^{+}=f_{0}$, but more importantly

$$
\mathcal{F}_{n}^{+}=\left(\Lambda_{0}^{-1} \circ \Lambda_{Q}\right) \circ\left(f_{Q-1} \circ \cdots \circ f_{j} \circ \cdots f_{1}\right) .
$$

We note also that for all $t \in \Delta_{n, j}^{+}$

$$
S f_{j}(t)=S f\left(\Lambda_{j}(t)\right)\left[D \Lambda_{j}(t)\right]^{2}=S f\left(\Lambda_{j}(t)\right)\left|I_{n-1}^{j}\right|^{2},
$$

by the chain rule for the Schwarzian derivative.

Notation. Given $J=[a, b] \subseteq \mathbb{R}$ and $\tau>0$, we denote by $J^{\tau}$ the interval $[c, d] \supseteq J$ such that $(a-c) /(b-a)=(d-b) /(b-a)=\tau$. Note that $J$ has space equal to $\tau$ inside $J^{\tau}$.

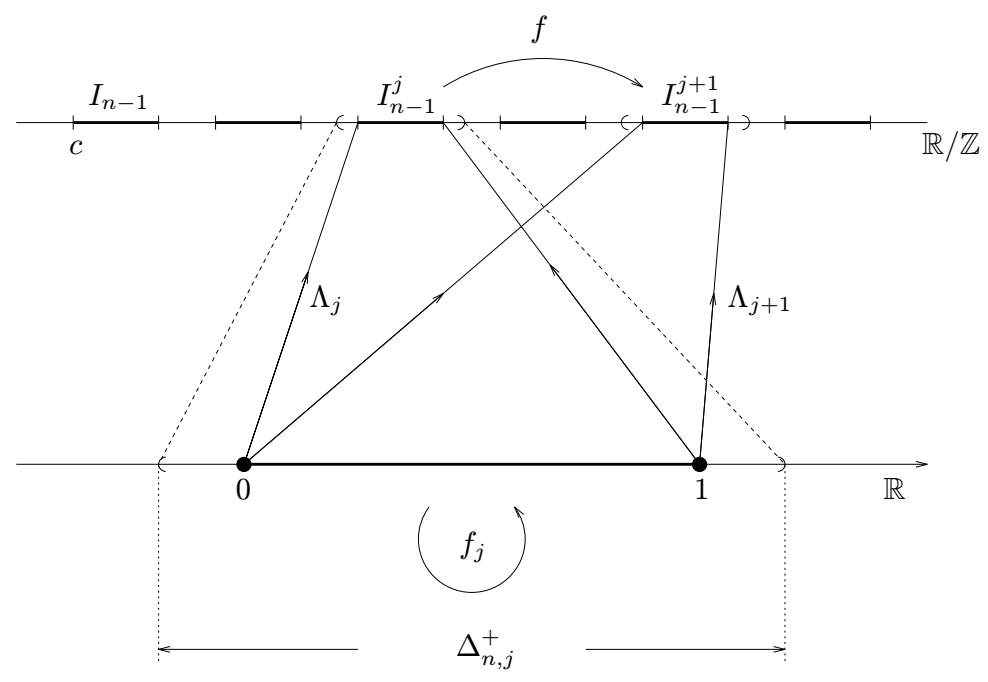

Fig. 5. The elementary factors of $\mathcal{F}_{n}^{+}$ 
Theorem A.4. (The $C^{2}$ bounds)

Let $f \in \mathrm{Crit}^{3}\left(S^{1}\right)$ be a critical circle map with arbitrary irrational rotation number, let $f_{n}:\left[\lambda_{n}, 1\right] \rightarrow \mathbb{R}$ be the $n$-th renormalization of $f$, and let $\mathcal{F}_{n}^{ \pm}$: $\Delta_{n}^{ \pm} \rightarrow \mathbb{R}$ be the coefficients of $f_{n}$. Also, let $f_{j}: \Delta_{n, j}^{+} \rightarrow \Delta_{n, j+1}^{+}$be the elementary factors of $\mathcal{F}_{n}^{+}$. There exist positive constants $B$ and $\tau$ depending only on the real bounds for $f$ such that the following statements hold for all $n \geq 1$.

(a) We have $\Delta_{n}^{-} \supseteq\left[\lambda_{n}, 0\right]^{\tau}$ and $\Delta_{n}^{+} \supseteq[0,1]^{\tau}=[-\tau, 1+\tau]$.

(b) For all $0 \leq j \leq Q$, we have $\Delta_{n, j}^{+} \supseteq[0,1]^{\tau}$.

(c) We have $\left|S \mathcal{F}_{n}^{-}(t)\right| \leq B$ for all $t \in \Delta_{n}^{-}$and $\left|S \mathcal{F}_{n}^{+}(t)\right| \leq B$ for all $t \in \Delta_{n}^{+}$.

(d) More generally, for all $1 \leq j<k \leq Q$, we have $\left|S\left(f_{k} \circ \cdots \circ f_{j}\right)(t)\right| \leq B$ for all $t \in \Delta_{n, j}^{+}$.

(e) The $C^{2}$ norms of the restrictions $\mathcal{F}_{n}^{-} \mid\left[\lambda_{n}, 0\right]^{\tau / 2}$ and $\mathcal{F}_{n}^{+} \mid[0,1]^{\tau / 2}$ are bounded by $B$.

(f) More generally, for all $1 \leq j<k \leq Q$, the $C^{2}$ norm of the restriction of $f_{k} \circ \cdots \circ f_{j}$ to the interval $f_{j-1} \circ \cdots \circ f_{1}\left([0,1]^{\tau / 2}\right)$ is bounded by $B$.

(g) The $C^{2}$ norms of $\boldsymbol{f}_{n}^{-}$and $\boldsymbol{f}_{n}^{+}$are bounded by $B$.

Moreover, if $n$ is sufficiently large then both coefficients have negative Schwarzian derivatives at all points of their respective domains.

The proof will use the following lemma concerning the dynamical partitions $\mathcal{P}_{n}$. Let us denote by $d(c, I)$ the distance between an interval $I \subseteq S^{1}$ and the critical point $c$. For each $n \geq 1$, let

$$
S_{n}=\sum_{I \in \mathcal{P}_{n} \backslash\left\{I_{n-1}, I_{n}\right\}}\left(\frac{|I|}{d(c, I)}\right)^{2} \text {. }
$$

Lemma A.5. The sequence $S_{n}$ is bounded (by a constant depending only on $f$ ).

Proof. Recall that $\mathcal{P}_{n+2}$ is a strict refinement of $\mathcal{P}_{n}$. From the real bounds, we know that there exists a constant $0<\lambda<1$ depending only on $f$ such that, if $I$ is in $\mathcal{P}_{n}$ and $J \subseteq I$ is in $\mathcal{P}_{n+2}$, then $|J| \leq \lambda|I|$. Hence

$$
\sum_{I \supseteq J \in \mathcal{P}_{n+2}}|J|^{2} \leq\left(\max _{I \supseteq J \in \mathcal{P}_{n+2}}|J|\right)|I| \leq \lambda|I|^{2} .
$$

Since we also have $d(c, J) \geq d(c, I)$ whenever $J \subseteq I$, it follows that

$$
\begin{gathered}
S_{n+2} \leq \lambda S_{n}+\sum_{\mathcal{P}_{n+2} \ni J \subseteq I_{n-1} \backslash I_{n+1}}\left(\frac{|J|}{d(c, J)}\right)^{2}+\sum_{\mathcal{P}_{n+2} \ni J \subseteq I_{n} \backslash I_{n+2}}\left(\frac{|J|}{d(c, J)}\right)^{2} \\
\leq \lambda S_{n}+\lambda\left(\frac{\left|I_{n-1}\right|}{\left|I_{n+1}\right|}\right)^{2}+\lambda\left(\frac{\left|I_{n}\right|}{\left|I_{n+2}\right|}\right)^{2} .
\end{gathered}
$$


From this and the facts that $\left|I_{n-1}\right| \asymp\left|I_{n+1}\right|$ and $\left|I_{n}\right| \asymp\left|I_{n+2}\right|$, we get $S_{n+2} \leq \lambda S_{n}+\mu$, where $\mu$ is a constant depending only on $f$. But then, by induction,

$$
S_{2 n} \leq \lambda^{n-1} S_{2}+\frac{\mu}{1-\lambda}, \quad S_{2 n+1} \leq \lambda^{n} S_{1}+\frac{\mu}{1-\lambda},
$$

and therefore $S_{n}$ is bounded as claimed.

Proof of Theorem A.4. It is enough to prove this theorem under the assumption that $f$ is canonical. The existence of $\tau>0$ such that $(a)$ and $(b)$ hold is a consequence of the real bounds. Hence we proceed to prove $(c)$ for $\mathcal{F}_{n}^{+}$, the proof for $\mathcal{F}_{n}^{-}$being completely similar. Making $\tau$ smaller if necessary and using the classical Koebe non-linearity principle, we can assume that there exists $C>0$ depending only on the real bounds for $f$ such that

$$
\left|D\left(f_{j} \cdots f_{1}\right)(t)\right| \leq C,
$$

for all $t \in[-\tau, 1+\tau]$ and all $j=1, \ldots, Q-1$.

Let $\mathcal{V} \subseteq S^{1}$ be an open set whose closure does not contain $c$ and such that $\mathcal{U} \cup \mathcal{V}=S^{1}$. Also, let $M=\sup _{x \in \mathcal{V}}|S f(x)|$. We assume that $n$ is so large that the largest interval in $\mathcal{P}_{n}$ has length smaller than the Lebesgue number of the covering $\{\mathcal{U}, \mathcal{V}\}$. Together with (37) and (38), iterated use of the chain rule for the Schwarzian yields

$$
\begin{aligned}
S \mathcal{F}_{n}^{+}(t) & =S\left(f_{Q-1} \cdots f_{j} \cdots f_{1}\right)(t) \\
& =\sum_{j=1}^{Q-1} S f_{j}\left(f_{j-1} \cdots f_{1}(t)\right)\left[D\left(f_{j-1} \cdots f_{1}\right)(t)\right]^{2} \\
& =\sum_{j=1}^{Q-1} S f\left(\Lambda_{j} f_{j-1} \cdots f_{1}(t)\right)\left|I_{n-1}^{j}\right|^{2}\left[D\left(f_{j-1} \cdots f_{1}\right)(t)\right]^{2} .
\end{aligned}
$$

We split this last sum into $\Sigma_{1}(t)+\Sigma_{2}(t)$, where $\Sigma_{1}(t)$ is the sum over all $j$ 's such that $I_{n-1}^{j} \subseteq \mathcal{U}$ and $\Sigma_{2}(t)$ is the sum over the remaining terms (i.e. those with $I_{n-1}^{j} \subseteq \mathcal{V}$ ). Then we have on one hand

$$
\left|\Sigma_{2}(t)\right| \leq C^{2} M \sum_{I_{n-1}^{j} \subseteq \mathcal{V}}\left|I_{n-1}^{j}\right|^{2} \leq C^{2} M \max _{1 \leq j \leq Q-1}\left|I_{n-1}^{j}\right| .
$$

On the other hand, since $d\left(c, J_{n, j}^{+}\right) \asymp d\left(c, I_{n-1}^{j}\right)$ for all $j$, we have by (36)

$$
\left|\Sigma_{1}(t)\right| \leq C^{2} \sum_{I_{n-1}^{j} \subseteq \mathcal{U}} \frac{\left|I_{n-1}^{j}\right|^{2}}{\left[d\left(c, J_{n, j}^{+}\right)\right]^{2}} \leq C^{\prime} S_{n},
$$


where $C^{\prime}$ is another constant depending only on $f$ and $S_{n}$ is given by (39). From (41) and (42) it follows that $\left|S \mathcal{F}_{n}^{+}(t)\right|$ is uniformly bounded, and this proves $(c)$. Moreover, since by (41) $\Sigma_{2}(t)$ goes to zero with $n$ while $\Sigma_{1}(t)$ is always negative and bounded away from zero, we deduce that $S \mathcal{F}_{n}^{+}(t)<0$ for all $n$ sufficiently large. The proof of $(d)$ is entirely analogous.

To prove $(e)$, let $B_{0}$ be the upper-bound that we have just obtained for $\left|S \mathcal{F}_{n}^{+}\right|$. Applying Lemma A.3 to $\mathcal{F}_{n}^{+}$, we get for all $t \in[0,1]^{\tau / 2}$

$$
\left|\frac{D^{2} \mathcal{F}_{n}^{+}(t)}{D \mathcal{F}_{n}^{+}(t)}\right| \leq K_{\tau_{0}, B_{0}}
$$

where $\tau_{0}=\tau / 2(1+\tau)$ is the space of $[0,1]^{\tau / 2}$ inside $[0,1]^{\tau}$. Therefore $\left\|D^{2} \mathcal{F}_{n}^{+}\right\|_{0} \leq K_{\tau_{0}, B_{0}}\left\|D \mathcal{F}_{n}^{+}\right\|_{0} \leq C K_{\tau_{0}, B_{0}}$, by (40) above. This shows that the $C^{2}$ norm of $\mathcal{F}_{n}^{+}$is bounded as claimed. A similar argument proves $(f)$. Finally, $(g)$ follows from $(e)$ and the fact that the folding factors $\varphi_{n}^{ \pm}$are linear blow-ups of a fixed power-law map. The theorem is therefore proved if we take $B$ to be the largest of all the upper-bounds obtained in the argument. $\square$

Remark. We can go a bit further in $(e),(f)$ and $(g)$ and bound also the $C^{3}$ norms. For this purpose, it suffices to note for instance that

$$
D^{3} \mathcal{F}_{n}^{+}(t)=D \mathcal{F}_{n}^{+}(t)\left(S \mathcal{F}_{n}^{+}(t)+\frac{3}{2}\left[\frac{D^{2} \mathcal{F}_{n}(t)}{D \mathcal{F}_{n}(t)}\right]^{2}\right),
$$

and then use $(c)$ and $(e)$. However, this argument does not generalize to get bounds for higher derivatives. Our bootstrap argument in the next section will follow a different route, based on the $C^{m}$ Approximation Lemma.

\section{A.4. Bounding the $C^{r-1}$ norms}

We will show that the sequence of renormalizations of a $C^{r}$ critical circle map is bounded in the $C^{r-1}$ sense. The limits fall into (a compact subset of) a special family of analytic critical commuting pairs known as the Epstein class. Moreover, we will prove that such limits are attained at an exponential rate in the $C^{r-1}$ topology. The rate of convergence turns out to depend only on the rotation number of the given critical circle map.

An Epstein map is a homeomorphism $\varphi: I \rightarrow J$ between closed intervals on the real line such that $\varphi^{-1}$ is the restriction of an analytic univalent map $\Phi: \mathbb{C}\left(J^{\prime}\right) \rightarrow \mathbb{C}\left(I^{\prime}\right)$, where $I^{\prime} \supseteq I$ and $J^{\prime} \supseteq J$ are open intervals. Here we use the notation $\mathbb{C}(\Delta)=(\mathbb{C} \backslash \mathbb{R}) \cup \Delta$. For example, every fractional linear transformation in $P S L_{2}(\mathbb{R})$ is an Epstein map when restricted to an interval on the line which does not contain any of its poles. Further examples include polynomial or rational diffeomorphisms with real coefficients. 
Definition. A commuting pair $\boldsymbol{f}$ is said to be an Epstein commuting pair if $\boldsymbol{f}^{+}=\varphi^{+} \circ Q$ and $\boldsymbol{f}^{-}=\varphi^{-} \circ Q$, where $\varphi^{+}, \varphi^{-}$are Epstein maps and $Q$ is the power-law map $x \mapsto x^{p}$ (for some $p>1$ ).

Theorem A.6. Let $r \geq 3$ and let $f$ be a $C^{r}$ critical circle map with arbitrary irrational rotation number. Then the sequence of renormalizations $\left\{\mathcal{R}^{n}(f)\right\}$ is bounded in the $C^{r-1}$ metric and converges $C^{r-1}$ exponentially fast to the Epstein class.

The idea behind the proof of Theorem A.6 is quite simple. In the long composition defining the $n$-th renormalization of a critical circle map, we replace the factors away from the critical point by suitable fractional linear approximations, which are all Epstein maps. The factors which are close to the critical point are already Epstein because the map is assumed to be a power-law there. Therefore the entire new composition is an Epstein map. The Moebius approximations have to be carefully chosen, however, so that the total error involved, estimated with the help of the $C^{m}$ Approximation Lemma, be exponentially small in $n$ (the step of renormalization). We now present the technical result which is needed.

Lemma A.7. Given $r \geq 3$ and an orientation preserving $C^{r}$-diffeomorphism $\phi: I \rightarrow \mathbb{R}$ of a closed interval I onto its image, there exist constants $\ell_{\phi}>0$ and $K_{\phi}>0$ with the following property. For each closed interval $\Delta \subseteq I$ of length $|\Delta| \leq \ell_{\phi}$, there exists a fractional linear transformation $T_{\Delta} \in P S L_{2}(\mathbb{R})$ with $T_{\Delta}(\Delta)=\phi(\Delta)$ such that,

(a) $\sup _{x \in \Delta}\left|D^{k} \phi(x)-D^{k} T_{\Delta}(x)\right| \leq K_{\phi}|\Delta|^{3-k}$ for $k=0,1,2$.

(b) $\sup _{x \in \Delta}\left|D^{k} T_{\Delta}(x)\right| \leq K_{\phi}$ for all $1 \leq k \leq r$.

Proof. Let $\ell_{\phi}$ be the constant

$$
\ell_{\phi}=\min \left\{1, \inf _{x \in I}\left|\frac{\phi^{\prime}(x)}{\phi^{\prime \prime}(x)}\right|\right\} .
$$

Take any closed interval $\Delta \subseteq I$ with $|\Delta| \leq \ell_{\phi}$, and let $x_{0}$ be the left endpoint of $\Delta$. Let $T$ be the unique fractional linear transformation with the same 2-jet as $\phi$ at $x_{0}$. Thus, if $T(x)=\left(a\left(x-x_{0}\right)+b\right) /\left(c\left(x-x_{0}\right)+d\right)$ with $a d-b c=1$, then the coefficients are uniquely determined by the conditions

$$
\begin{aligned}
& T\left(x_{0}\right)=\frac{b}{d}=\phi\left(x_{0}\right), \\
& T^{\prime}\left(x_{0}\right)=\frac{1}{d^{2}}=\phi^{\prime}\left(x_{0}\right), \\
& T^{\prime \prime}\left(x_{0}\right)=\frac{-2 c}{d^{3}}=\phi^{\prime \prime}\left(x_{0}\right) .
\end{aligned}
$$


Moreover, for all $k \geq 1$,

$$
D^{k} T(x)=\frac{(-1)^{k+1} k ! c^{k-1}}{\left[c\left(x-x_{0}\right)+d\right]^{k+1}} .
$$

Since $\left|x-x_{0}\right| \leq|\Delta| \leq \ell_{\phi} \leq\left|\phi^{\prime}\left(x_{0}\right)\right| /\left|\phi^{\prime \prime}\left(x_{0}\right)\right|=|d| / 2|c|$ for all $x \in \Delta$, we have

$$
\frac{1}{2}|d| \leq\left|c\left(x-x_{0}\right)+d\right| \leq \frac{3}{2}|d|
$$

for each such $x$. Combining (44) with the lower bound in (45), we get

$$
\left|D^{k} T(x)\right| \leq \frac{2^{k+1} k !|c|^{k-1}}{|d|^{k+1}}=\frac{4 k !\left|\phi^{\prime \prime}\left(x_{0}\right)\right|^{k-1}}{\left|\phi^{\prime}\left(x_{0}\right)\right|^{k-2}},
$$

for all $x \in \Delta$ and all $k \geq 1$, and consequently

$$
\sup _{x \in \Delta}\left|D^{k} T(x)\right| \leq C_{0}=\max _{1 \leq k \leq r} \sup _{x \in I}\left\{\frac{4 k !\left|\phi^{\prime \prime}(x)\right|^{k-1}}{\left|\phi^{\prime}(x)\right|^{k-2}}\right\},
$$

when $1 \leq k \leq r$. In particular, from

$$
D^{2} \phi(x)-D^{2} T(x)=\int_{x_{0}}^{x} D^{3} \phi(t) d t-\int_{x_{0}}^{x} D^{3} T(t) d t,
$$

we deduce that

$$
\begin{aligned}
\left|D^{2} \phi(x)-D^{2} T(x)\right| & \leq\left\|D^{3} \phi\right\|_{0}\left|x-x_{0}\right|+\frac{24\left|\phi^{\prime \prime}\left(x_{0}\right)\right|^{2}}{\left|\phi^{\prime}\left(x_{0}\right)\right|}\left|x-x_{0}\right| \\
& \leq\left(\left\|D^{3} \phi\right\|_{0}+C_{0}\right)|\Delta|,
\end{aligned}
$$

for all $x \in \Delta$. Integrating this inequality twice, using (43), we get

$$
\sup _{x \in \Delta}\left|D^{k} \phi(x)-D^{k} T(x)\right| \leq C_{1}|\Delta|^{3-k},
$$

for $k=0,1,2$, where $C_{1}=C_{0}+\left\|D^{3} \phi\right\|_{0}$.

Looking at (46) and (47), we see that $T$ is almost what we want, but not quite because in general it does not map $\Delta$ onto $\phi(\Delta)$. To correct this flaw, we replace $T$ by $T_{\Delta}=A \circ T$, where $A$ is the unique affine, orientationpreserving map that carries $T(\Delta)$ onto $\phi(\Delta)$. We have

$$
A(t)-t=\left[\frac{|\phi(\Delta)|}{|T(\Delta)|}-1\right]\left(t-T\left(x_{0}\right)\right),
$$

for all $t \in T(\Delta)$, because $\phi\left(x_{0}\right)=T\left(x_{0}\right)$. Let $\mu=|\phi(\Delta)| /|T(\Delta)|$. Since by (47) we have ||$\phi(\Delta)|-| T(\Delta)|| \leq 2 C_{1}|\Delta|^{3}$, and since by the upper-bound in (45) we have

$$
\frac{|T(\Delta)|}{|\Delta|} \geq \inf _{x \in \Delta} \frac{1}{\left[c\left(x-x_{0}\right)+d\right]^{2}} \geq \frac{4}{9 d^{2}}=\frac{4}{9}\left|\phi^{\prime}\left(x_{0}\right)\right|,
$$


it follows that

$$
|\mu-1| \leq \frac{9 C_{1}}{2\left|\phi^{\prime}\left(x_{0}\right)\right|}|\Delta|^{2} .
$$

Thus we see that, for all $t \in T(I)$,

$$
\left|A^{\prime}(t)-1\right|=|\mu-1| \leq \frac{9 C_{1}}{2 \inf _{x \in I}\left|\phi^{\prime}(x)\right|}|\Delta|^{2}=C_{2}|\Delta|^{2},
$$

On the other hand, since $|T(\Delta)| \leq\|D \phi\|_{0}|\Delta|+2 C_{1}|\Delta|^{3}$, and since $|\Delta| \leq 1$, it follows from (48) that

$$
|A(t)-t| \leq C_{2}\left(\|D \phi\|_{0}+2 C_{1}\right)|\Delta|^{3}=C_{3}|\Delta|^{3} .
$$

Therefore

$$
\left|\phi(x)-T_{\Delta}(x)\right| \leq|\phi(x)-T(x)|+|T(x)-A(T(x))| \leq\left(C_{1}+C_{3}\right)|\Delta|^{3},
$$

and moreover, using the fact that $D^{k} T_{\Delta}(x)=\mu D^{k} T(x)$ for all $k$,

$$
\begin{aligned}
\left|D^{k} \phi(x)-D^{k} T_{\Delta}(x)\right| & \leq\left|D^{k} \phi(x)-D^{k} T(x)\right|+|\mu-1|\left|D^{k} T(x)\right| \\
& \leq C_{1}|\Delta|^{3-k}+C_{0} C_{2}|\Delta|^{2} \\
& \leq\left(C_{1}+C_{0} C_{2}\right)|\Delta|^{3-k},
\end{aligned}
$$

for all $x \in \Delta$ and $k=1,2$. Finally, for all $k \geq 1$ we have

$$
\left|D^{k} T_{\Delta}(x)\right| \leq\left(1+C_{2}|\Delta|^{2}\right)\left|D^{k} T(x)\right| \leq\left(1+C_{2}\right) C_{0} .
$$

Part (a) now follows from (49) and (50), while part (b) follows from (51), provided we take $K_{\phi}=\max \left\{C_{1}+C_{3}, C_{1}+C_{0} C_{2},\left(1+C_{2}\right) C_{0}\right\}$.

Proof of Theorem A.6. We now expand the outline given above and present a complete proof of Theorem A.6. In the proof, we will denote by $C_{0}, C_{1}, \ldots$ positive constants depending only on the real bounds for $f$. As before, we may assume from the start that $f$ is canonical, and accordingly we consider the covering $\{\mathcal{U}, \mathcal{V}\}$ of $S^{1}$ defined in the proof of Theorem A.4. Since the folding factors of $\boldsymbol{f}_{n}$ are power-law maps, and therefore already Epstein, it suffices to prove that the coefficients of $\boldsymbol{f}_{n}$ can be approximated by Epstein maps, up to an error exponentially small in $n$ in the $C^{r-1}$ topology. We will do this for $\mathcal{F}_{n}^{+}$, the proof for $\mathcal{F}_{n}^{-}$being the same.

As in the previous section, let $f_{j}: \Delta_{n, j}^{+} \rightarrow \Delta_{n, j+1}^{+}, 1 \leq j \leq Q-1$, be the elementary factors of $\mathcal{F}_{n}^{+}$. For each $1 \leq j \leq Q$ we define

$$
\Delta_{j}=f_{j-1} \circ \cdots \circ f_{2} \circ f_{1}\left([0,1]^{\tau / 4}\right) \subseteq \Delta_{n, j}^{+},
$$

where $\tau$ is the constant of Theorem A.4. Note that $f_{j}\left(\Delta_{j}\right)=\Delta_{j+1}$. Let $\Delta_{j}^{\prime}=\Lambda_{j}\left(\Delta_{j}\right)$, and observe also that $I_{n-1}^{j} \subseteq \Delta_{j}^{\prime} \subseteq J_{n, j}^{+}$. 
We introduce individual Epstein approximations $g_{j}$ to each $f_{j}$. There are two cases to consider. It may happen that $\Delta_{j}^{\prime} \subseteq \mathcal{U}$, in which case we simply take $g_{j}=f_{j}$. Otherwise, we have $\Delta_{j}^{\prime} \subseteq \mathcal{V}$. In this case, we let $T_{j}: \Delta_{j}^{\prime} \rightarrow \Delta_{j+1}^{\prime}$ be the Moebius approximation to $f \mid \Delta_{j}^{\prime}$ that we get applying Lemma A.7 to the restriction of $f$ to $\mathcal{V}$, and then take $g_{j}=\Lambda_{j+1}^{-1} \circ T_{j} \circ \Lambda_{j}$. Note that $g_{j}\left(\Delta_{j}\right)=\Delta_{j+1}$.

Claim 1. We have $\left\|f_{j}-g_{j}\right\|_{r} \leq C_{0}\left|I_{n-1}^{j}\right|^{2}$ for all $j$.

This is obvious when $I_{n-1}^{j} \subseteq \mathcal{U}$. When $I_{n-1}^{j} \subseteq \mathcal{V}$, we have $\left|I_{n-1}^{j}\right| \asymp$ $\left|I_{n-1}^{j+1}\right|$, because the derivative of $f$ on $\mathcal{V}$ is bounded away from zero, and we also have $\left|\Delta_{j}^{\prime}\right| \asymp\left|I_{n-1}^{j}\right|$. Moreover, for all $1 \leq s \leq r$ and all $x \in \Delta_{j}$,

$$
D^{s} f_{j}(x)-D^{s} g_{j}(x)=\frac{\left|I_{n-1}^{j}\right|^{s}}{\left|I_{n-1}^{j+1}\right|}\left(D^{s} f\left(\Lambda_{j}(x)\right)-D^{s} T_{j}\left(\Lambda_{j}(x)\right)\right) \text {. }
$$

Therefore the claim follows from Lemma A.7 (treat the cases $s=1,2$ separately).

Now, recall from Theorem A.4 that for all $1 \leq j<k \leq Q-1$ we have

$$
\left\|f_{k} \circ \cdots \circ f_{j}\right\|_{2} \leq B \text {. }
$$

Claim 2. If $n$ is sufficiently large then for all $1 \leq j<k \leq Q-1$ we have

$$
\left\|f_{k} \circ \cdots \circ f_{j}-g_{k} \circ \cdots \circ g_{j}\right\|_{1} \leq C_{1} \max _{0 \leq i \leq Q}\left|I_{n-1}^{i}\right| .
$$

Take $n_{0}$ so large that $C_{0} \max \left|I_{n_{0}-1}^{j}\right|<\varepsilon_{B}$, where $\varepsilon_{B}$ is the constant given by Lemma A. 2 when we take $M=B$. Then from Claim 1 and (52), the hypotheses of Lemma A.2 are satisfied, and we get for all $n \geq n_{0}$

$$
\begin{aligned}
\left\|f_{k} \circ \cdots \circ f_{j}-g_{k} \circ \cdots \circ g_{j}\right\|_{1} & \leq C_{B} \sum_{i=j}^{k}\left\|f_{i}-g_{i}\right\|_{2} \leq C_{0} C_{B} \sum_{i=j}^{k}\left|I_{n-1}^{i}\right|^{2} \\
& \leq C_{0} C_{B} \max _{0 \leq i \leq Q}\left|I_{n-1}^{i}\right|,
\end{aligned}
$$

where $C_{B}$ is the constant of Lemma A.2 for $M=B$. This proves the claim.

In order to bootstrap these $C^{1}$ estimates up to $C^{r-1}$ estimates, we apply the $C^{m}$ Approximation Lemma once more, this time reversing the roles of $f_{j}$ and $g_{j}$, and with $m=r$. Thus, we need to verify the hypotheses of that lemma in this new situation.

Claim 3. For all $1 \leq j<k \leq Q-1$, we have $\left\|g_{k} \circ \cdots \circ g_{j}\right\|_{r} \leq C_{2}$.

For brevity, write $G_{j k}=g_{k} \circ \cdots \circ g_{j}$. Then $G_{j k}^{-1}$ is univalent on $\mathbb{C}\left(\Delta_{j k}\right)$, where $\Delta_{j k}$ is an interval containing $G_{j k}\left(\Delta_{j}\right)$ with definite space on both 
sides, by our choice of $\tau$. Using Koebe's one-quarter theorem, it is not difficult to see that the domain $\Omega_{j k}=G_{j k}^{-1}\left(\mathbb{C}\left(\Delta_{j k}\right)\right)$ contains a rectangle $W_{j}=\Delta_{j}^{\alpha} \times[-\beta, \beta]$, and that $d\left(\partial W_{j}, \partial \Omega_{j k}\right) \geq \gamma$ where $\alpha, \beta$ and $\gamma$ are positive constants depending only on $\tau$ and the real bounds for $f$. Hence, from the complex Koebe's distortion theorem, we get

$$
\left|\frac{G_{j k}^{\prime}(z)}{G_{j k}^{\prime}(w)}\right| \leq \exp \left\{\frac{4}{\gamma} \operatorname{diam}\left(W_{j}\right)\right\} \leq C_{3},
$$

for all $z, w \in W_{j}$. This together with the mean-value theorem gives us $\left|G_{j k}^{\prime}(z)\right| \leq C_{4}$, and therefore also $\left|G_{j k}(z)\right| \leq C_{5}$, for all $x \in W_{j}$. Now we use Cauchy's integral formula to bound all higher derivatives of $G_{j k}$. We have for all $x \in \Delta_{j}$ and all $s \geq 1$

$\left|D^{s} G_{j k}(x)\right|=\frac{s !}{2 \pi}\left|\int_{\partial W_{j}} \frac{G_{j k}(z)}{(z-x)^{s+1}} d z\right| \leq \frac{C_{5} s !}{\pi}\left(\beta+(1+2 \alpha)\left|\Delta_{j}\right|\right) \delta_{j}^{-s-1}$,

where $\delta_{j}=\inf _{x \in \Delta_{j}} d\left(x, \partial W_{j}\right)=\min \left\{\alpha\left|\Delta_{j}\right|, \beta\right\} \geq \delta=\min \{a, b\}$. Therefore $\left|D^{s} G_{j k}(x)\right| \leq C_{6} s ! \delta^{-s-1}$. This shows that $\left\|G_{j k}\right\|_{r}$ is bounded as claimed.

From Claims 1 and 3, the hypotheses of Lemma A.2 are therefore satisfied, and we have

$$
\begin{aligned}
\left\|f_{k} \circ \cdots \circ f_{j}-g_{k} \circ \cdots \circ g_{j}\right\|_{r-1} & \leq C_{C_{2}} \sum_{i=j}^{k}\left\|f_{i}-g_{i}\right\|_{r} \\
& \leq C_{0} C_{C_{2}} \sum_{i=j}^{k}\left|I_{n-1}^{i}\right|^{2} \\
& \leq C_{0} C_{C_{2}} \max _{0 \leq i \leq Q}\left|I_{n-1}^{i}\right|,
\end{aligned}
$$

this time for all $n$ large enough so that $C_{0} \max \left|I_{n-1}^{j}\right|<\varepsilon_{C_{2}}$, where $C_{C_{2}}$ and $\varepsilon_{C_{2}}$ are the constants of Lemma A.2 for $M=C_{2}$. Since max $\left|I_{n-1}^{j}\right|$ decreases exponentially with $n$, we are done.

\section{Appendix B. Proof of Yoccoz's Lemma}

The main geometric idea behind the proof of Yoccoz's Lemma is to use the negative Schwarzian property of $f$ to squeeze the graph of $f$ between the graphs of two Moebius transformations. The required estimate for $f$ will then follow from the corresponding estimate for Moebius transformations, which we now state and prove. 
Consider the fractional linear transformation $T(x)=x /(1+x)$, and given $\varepsilon>0$, let $T_{\varepsilon}(x)=T(x)-\varepsilon$. We are interested in certain quantitative aspects of the orbit $x_{n}=T_{\varepsilon}^{n}\left(x_{0}\right)$ for $x_{0}=1$. Observe that this sequence is strictly decreasing.

Lemma B.1. Let $N>0$ be such that $x_{N+1} \leq 0<x_{N}$. Then we have $N \asymp 1 / \sqrt{\varepsilon}$ and moreover $x_{n}-x_{n+1} \asymp 1 / n^{2}$ for $n=0,1, \ldots, N$.

Proof. Writing $\delta_{n}=T^{n}\left(x_{0}\right)-T_{\varepsilon}^{n}\left(x_{0}\right)$, we have

$$
\delta_{n}=\varepsilon+\frac{\delta_{n-1}}{\left(1+\frac{1}{n}\right)\left(1+\frac{1}{n}-\delta_{n-1}\right)}
$$

for all $n=1,2, \ldots, N+1$. We claim that

$$
\frac{n \varepsilon}{6} \leq \delta_{n} \leq n \varepsilon
$$

The last inequality is clear. To prove the first, we note from (53) that

$$
\delta_{n} \geq \varepsilon+\left(\frac{n}{n+1}\right)^{2} \delta_{n-1} .
$$

By induction, this gives us

$$
\begin{aligned}
\delta_{n} \geq \frac{\varepsilon}{(n+1)^{2}}\left(1^{2}+2^{2}+\cdots+n^{2}\right) & =\frac{\varepsilon}{(n+1)^{2}} \frac{n(n+1)(2 n+1)}{6} \\
& \geq \frac{n \varepsilon}{6},
\end{aligned}
$$

which proves the claim. Now, from the fact that $x_{N+1} \leq 0<x_{N}$ we have the inequalities

$$
\delta_{N}<\frac{1}{N+1}, \quad \delta_{N+1} \geq \frac{1}{N+2} .
$$

Then, using (54), we get

$$
\frac{1}{(N+1)(N+2)} \leq \varepsilon<\frac{6}{N(N+1)},
$$

which proves the first assertion.

Next, note that since $\left[x_{N+1}, x_{N}\right] \subseteq\left[T_{\varepsilon}(0), T_{\varepsilon}^{-1}(0)\right]=[-\varepsilon, \varepsilon /(1-\varepsilon)]$, we have

$$
\varepsilon<x_{N}-x_{N+1}<3 \varepsilon
$$

Hence, by (55), we get $x_{N}-x_{N+1} \asymp 1 / N^{2}$ and the second assertion is proved when $n=N$. To prove it in general using this information, observe that

$$
x_{n}-x_{n+1}=\frac{x_{n-1}-x_{n}}{\left(1+x_{n-1}\right)\left(1+x_{n}\right)}=\frac{x_{n-1}-x_{n}}{\left(1+\frac{1}{n}-\delta_{n-1}\right)\left(1+\frac{1}{n+1}-\delta_{n}\right)}
$$


implies

$$
x_{n}-x_{n+1} \geq \frac{n}{n+2}\left(x_{n-1}-x_{n}\right) .
$$

By induction, this gives on one hand

$$
x_{n}-x_{n+1} \geq \frac{2}{(n+1)(n+2)}\left(x_{0}-x_{1}\right) \geq \frac{1}{(n+1)(n+2)},
$$

and on the other hand, using (55) and (56),

$$
x_{n}-x_{n+1} \leq\left(x_{N}-x_{N+1}\right) \prod_{j=1}^{N-n}\left(\frac{n+j+2}{n+j}\right)<\frac{54}{(n+1)(n+2)} .
$$

This proves the second assertion in all cases.

Now recall that $f: \Delta_{1} \cup \Delta_{2} \cup \cdots \cup \Delta_{a} \rightarrow \mathbb{R}$ satisfies $f\left(\Delta_{j}\right)=\Delta_{j+1}$ for all $j$. Without loss of generality, we can assume that $f(x)<x$ for all $x$. Thus, if we call $x_{0}$ the right endpoint of $\Delta_{1}$ and write $x_{j}=f^{j}\left(x_{0}\right)$, we have $\Delta_{j}=\left[x_{j}, x_{j-1}\right]$ for all $j$. Since our map $f$ is a negative-Schwarzian diffeomorphism, there exists a unique $z$ in the domain of $f$ such that $\varepsilon=|f(z)-z| \leq|f(x)-x|$ for all $x$. Since the statement we want to prove is invariant under affine changes of coordinates, we may assume also that $z=0$ and $x_{0}=1$. In this setting, we want to prove that $\left|\Delta_{j}\right| \asymp 1 / j^{2}$ for all $j$ such that $\Delta_{j} \subseteq[0,1]$. Note that $f^{\prime}(0)=1$.

Next, let $A$ be the Moebius transformation on the line such that $A\left(x_{0}\right)=f\left(x_{0}\right)$ and $A(0)=f(0)$ and $A^{\prime}(0)=f^{\prime}(0)=1$. This determines $A$ uniquely, and in fact

$$
A(x)=\frac{x}{1+\lambda x}-\varepsilon,
$$

for some $\lambda>0$. Since $S f<0$, we see that $A(x) \leq f(x)$ for all $x \in[0,1]$.

Likewise, let $B$ be the Moebius transformation such that $B\left(x_{a}\right)=f\left(x_{a}\right)$, $B(0)=f(0)$ and $B^{\prime}(0)=f^{\prime}(0)=1$. This determines $B$ uniquely, and in fact

$$
B(x)=\frac{x}{1+\mu x}-\varepsilon,
$$

for some $\mu>0$. This time, since $x_{a}<0$ and $S f<0$, we have $f(x) \leq B(x)$ for all $x \in[0,1]$. In particular, $\lambda>\mu$. It is easy to see that $\lambda / \mu \leq c_{\sigma}$, where $c_{\sigma}$ depends only on the constant $\sigma$ in the statement.

Lemma B.2. Let $x \in[0,1]$ and $k>0$ be such that $A(x)<B^{k}(x)$. Then $k \leq 1+\lambda / \mu$. 
Proof. By induction we have

$$
B^{k}(x) \leq \frac{x}{1+(k-1) \mu x}-\varepsilon .
$$

Therefore $A(x)<B^{k}(x)$ implies $(k-1) \mu x<\lambda x$.

Now, let us write $\alpha_{n}=A^{n}\left(x_{0}\right)$ and $\beta_{n}=B^{n}\left(x_{0}\right)$. By Lemma B.2, the number of $\beta_{j}$ 's inside each interval of the form $\left[\alpha_{n+1}, \alpha_{n}\right]$ is bounded independently of $n$. Moreover, since $\alpha_{n}<x_{n}<\beta_{n}$ for all $n$, the number of $x_{j}$ 's inside each $\left[\alpha_{n+1}, \alpha_{n}\right]$ is also bounded independently of $n$. To prove that $\left|\Delta_{j}\right| \asymp 1 / j^{2}$, we proceed as follows. Let $\ell>0$ be such that $\beta_{\ell+1} \leq x_{j} \leq \beta_{\ell} \leq x_{j-1}$. Then Lemma B.2 says that $\ell \leq C j$, and we have also

$$
\left|\beta_{\ell+1}-\beta_{\ell}\right|<\left|B\left(x_{j-1}\right)-x_{j-1}\right|<\left|x_{j}-x_{j-1}\right| .
$$

Since by Lemma B.1 we have

$$
\left|\beta_{\ell+1}-\beta_{\ell}\right| \asymp \frac{1}{\ell^{2}} \geq \frac{1}{C j^{2}},
$$

it follows that $\left|\Delta_{j}\right|=\left|x_{j}-x_{j-1}\right| \geq 1 / C j^{2}$.

To prove an inequality in the opposite direction, let $m$ be the largest integer such that $\alpha_{m}>x_{j-1}$. Then, again by Lemma B.2, we have $j \leq C m$. Since $A(x)<f(x)<x$ for all $x$, we also have $\Delta_{j} \subseteq\left[\alpha_{m+2}, \alpha_{m}\right]$. Using Lemma B.1 once more, we deduce that

$$
\left|\Delta_{j}\right| \leq \frac{C}{m^{2}} \leq \frac{C}{j^{2}} .
$$

This completes the proof of Yoccoz's Lemma.

\section{Appendix C. A full-measure condition on rotation numbers}

We present here our account of C.G. Moreira's probabilistic argument showing that condition (12) holds for a set of full-measure in [0,1]. His original probability estimates were done for Lebesgue measure in $[0,1]$. We prefer to use instead a probability measure which is invariant under the Gauss map, namely the Gauss measure. These two measures are mutually absolutely continuous, the density of the latter with respect to the former being $d x /(1+x) \log 2$. With respect to the Gauss probability measure (the only one we will use from now on), the partial quotients $a_{n}(\theta)$ of $\theta \in[0,1]$ are identically distributed random variables. We warn the reader that these are not independent random variables; they are only asymptotically independent, a fact that is perhaps best expressed by saying that the Gauss map is mixing. 
Now, the probability that the $n$-th partial quotient $a_{n}(\theta)$ be equal to a given integer $m \geq 1$ is

$$
p_{m}=\mathbb{P}\left[a_{n}(\theta)=m\right]=\frac{1}{\log 2} \log \left(1+\frac{1}{m(m+2)}\right)<\frac{2}{m^{2}} .
$$

From this, we see that the probability that $a_{n}(\theta)$ be at least $m$ is smaller than $4 / m$. These facts form the basis for our estimates, and yield the following lemma.

Lemma C.1. There exists a full-measure set $E \subseteq[0,1]$ such that for each $\theta \in E$ we have $a_{n}(\theta)<n^{2}$ for all sufficiently large $n$.

Proof. Let $E=\liminf \left([0,1] \backslash A_{n}\right)$, where $A_{n}$ is the set of all $\theta \in[0,1]$ such that $a_{n}(\theta) \geq n^{2}$. Then $\mathbb{P}\left(A_{n}\right)<4 / n^{2}$, and since the series $\sum 4 / n^{2}$ converges, we deduce by the Borel-Cantelli lemma that $\mathbb{P}(E)=1$.

We shall prove the following result, which establishes condition (12) for almost all numbers $\theta \in[0,1]$ with $\omega(t)=C_{\theta}(1-\log t)$, where $C_{\theta}>0$.

Proposition C.2. Almost all numbers $\theta \in E$ satisfy, for all $1 \leq n \leq k$,

$$
\frac{1}{n} \sum_{j=k+1}^{k+n} \log a_{j}(\theta) \leq C_{\theta}\left(1+\log \frac{k}{n}\right),
$$

for some constant $C_{\theta}>0$.

Proof. In attempting to prove the inequality (58) for a given $\theta \in E$, we may assume that $k$ is so large that $a_{j}(\theta)<j^{2}$ for all $j \geq k$. The remaining cases, corresponding to the remaining finitely many pairs $(n, k)$, are taken care of by a suitable choice of the constant $C_{\theta}$.

Given $(n, k)$, there are two possibilities to consider. The first possibility is that $n^{2}<k$. In this case we simply observe that

$$
\frac{1}{n} \sum_{j=k+1}^{k+n} \log a_{j}(\theta) \leq 2 \log (k+n)<5 \log \frac{k}{n},
$$

for all sufficiently large $k$.

The second possibility is that $n \leq k \leq n^{2}$. Here, we shall prove that with probability one the left-hand side of (58) is bounded by 10. For this purpose, let us consider the following pathologies.

(a) For a given $m \geq 1$, there are more than $2 n p_{m}$ partial quotients $a_{k+i}(\theta)$ with $1 \leq i \leq n$ such that $a_{k+i}(\theta)=m$ (where $p_{m}$ is as defined in (57)). 
By an elementary combinatorial argument, we see that this occurs with probability at most

$$
\sum_{j=\left\lceil 2 n p_{m}\right\rceil}^{n}\left(\begin{array}{l}
n \\
j
\end{array}\right) p_{m}^{j}\left(1-p_{m}\right)^{n-j}<\left(\frac{e}{4}\right)^{n p_{m}} .
$$

The probability that this happens for some $m$ in the range $1 \leq m \leq n^{1 / 3}$ is therefore smaller than

$$
n^{1 / 3} \times\left(\frac{e}{4}\right)^{2 n^{1 / 3}}<\frac{1}{n^{4}}
$$

if $n$ is sufficiently large.

(b) There are more than $n^{2 / 3}$ partial quotients $a_{k+i}(\theta)$ with $1 \leq i \leq n$ such that $a_{k+i}(\theta)>n^{1 / 3}$. By a similar reasoning to the one used in $(a)$, we see that this occurs with probability smaller than

$$
\left(\frac{e}{4}\right)^{n^{1 / 3}}<\frac{1}{n^{4}}
$$

if $n$ is sufficiently large.

Therefore, fixing $n$ sufficiently large, the probability that there exists $k$ in the range $n \leq k \leq n^{2}$ such that one of the above pathologies occurs for $(n, k)$ is certainly less than $n^{2} \times\left(2 / n^{4}\right)=2 / n^{2}$. Since the series $\sum 2 / n^{2}$ converges, again by Borel-Cantelli we deduce that with probability one there are no pathologies for $(n, k)$ if $k$ (and hence $n$ ) is sufficiently large.

Now, if there are no pathologies for $(n, k)$, and noting that for $1 \leq i \leq n$ we have

$$
a_{k+i}(\theta)<(k+i)^{2} \leq\left(n^{2}+n\right)^{2} \leq 4 n^{4}
$$

if $k$ is sufficiently large, we deduce that

$$
\begin{aligned}
\frac{1}{n} \sum_{j=k+1}^{k+n} \log a_{j}(\theta) & \leq \frac{1}{n} \sum_{m=1}^{\left\lfloor n^{1 / 3}\right\rfloor}\left(2 n p_{m}\right) \log m+\frac{n^{2 / 3}}{n} \log \left(4 n^{4}\right) \\
& <\sum_{m=1}^{\left\lfloor n^{1 / 3}\right\rfloor} \frac{4 \log m}{m^{2}}+\frac{1}{n^{1 / 3}}(2 \log 2+4 \log n),
\end{aligned}
$$

which is less than 10 if $n$ is sufficiently large. This completes the proof.

Acknowledgements. We are grateful to Dennis Sullivan for suggesting many interesting ideas, and to Curt McMullen for explaining to us various aspects of his beautiful work on renormalization and rigidity. We want to thank the referee for very keen remarks leading to a considerable improvement of our final results. We also want to thank Jean-Christophe Yoccoz for providing us with a sketch of the proof of his unpublished theorem on quasisymmetric conjugacies between critical circle maps. We are indebted also to Marco Martens, 
Jacek Graczyk, Oscar Lanford, Rafael de la Llave, Michael Lyubich, Henri Epstein and C.G. Moreira for several useful discussions on these and related matters. This work has been financially sponsored by PRONEX, CNPq and FAPESP. We also would like to thank IHES, MSRI, ETH-Zürich, IMPA and SUNY at Stony Brook for their warm hospitality and generous support.

\section{References}

1. P. Billingsley: Probability and measure. New York: John Wiley \& Sons 1986

2. L. Carleson: On mappings conformal at the boundary. J. Anal. Math. 19, 1-13 (1967)

3. I. Cornfeld, S. Fomin, Ya. Sinai: Ergodic Theory. New York: Springer 1982

4. E. de Faria: Asymptotic rigidity of scaling ratios for critical circle mappings. Ergod. Th. \& Dynam. Sys. 19(4), 995-1035 (1999)

5. E. de Faria, W. de Melo: Rigidity of critical circle mappings II. To appear in J. Amer. Math. Soc., IMS Stony Brook preprint 97/17 (1997)

6. J. Franks: Manifolds of $C^{r}$ mappings and applications to differentiable dynamics. Stud. in Anal. Adv. Math. Suppl. Stud. 4, 271-290 (1979)

7. I. J. Good: The fractional dimension theory of continued fractions. Proc. Cambridge Phil. Soc. 37, 199-228 (1941)

8. J. Graczyk: Harmonic scalings for smooth families of diffeomorphisms of the circle. Nonlinearity 4, 935-954 (1990)

9. J. Graczyk: Hölder classes for circle maps. Proc. Amer. Math. Soc. (to appear)

10. J. Graczyk, G. Świa̧tek: Critical circle maps near bifurcation. Commun. Math. Phys. 176, 227-260 (1996)

11. M. Herman: Sur la conjugaison differentiable des difféomorphismes du cercle a des rotations. Publ. Math. IHES 49, 5-234 (1979)

12. M. Herman: Conjugaison quasi-simétrique des homéomorphismes du cercle a des rotations. 1988 (manuscript)

13. O. Lanford: Renormalization group methods for critical circle mappings with general rotation number. VIIIth International Congress on Mathematical Physics, pp.532-536 (Marseille, 1986). Singapore: World Sci. Publishing 1987

14. M. Lyubich: Combinatorics, geometry and attractors of quasi-quadratic mappings. Ann. of Math. 140, 347-404 (1994)

15. C. McMullen: Renormalization and 3-manifolds which fiber over the circle. Annals of Math Studies 142. Princeton: Princeton University Press 1996

16. W. de Melo, S. van Strien: One dimensional dynamics. Berlin, New York: Springer 1993

17. D. Rand: Global phase-space universality, smooth conjugacies and renormalization: the $C^{1+\alpha}$ case. Nonlinearity $\mathbf{1}, 181-202$ (1988)

18. G. Świątek: Rational rotation numbers for maps of the circle. Commun. Math. Phys. 119, 109-128 (1988)

19. G. Świątek: One-dimensional maps and Poincaré metric. Nonlinearity 4, 81-108 (1992)

20. J.C. Yoccoz: Conjugaison differentiable des diffeomorphismes du cercle dont le nombre de rotation verifie une condition Diophantienne. Ann. Sci. de l'Ec. Norm. Sup. 17, 333361 (1984)

21. J.C. Yoccoz: Il n'y a pas de contre-example de Denjoy analytique. C. R. Acad. Sci. Paris 298, 141-144 (1984) 